\title{
1 Organization of cortical and thalamic input to inhibitory neurons in mouse motor cortex
}

2

\section{1} 2

Sandra U. Okoro ${ }^{1}$, Roman U. Goz ${ }^{1}$, Brigdet W. Njeri ${ }^{1}$, Madhumita Harish ${ }^{1}$, Catherine F. Ruff ${ }^{1}$, Sarah E. Ross ${ }^{1}$, Charles R. Gerfen², Bryan M. Hooks ${ }^{1}$

${ }^{1}$ University of Pittsburgh School of Medicine, Pittsburgh, Pennsylvania, USA

${ }^{2}$ National Institute of Mental Health, Bethesda, Maryland, USA

Correspondence: Dr. Bryan M. Hooks, 200 Lothrop Street BSTWR Suite W1458

Pittsburgh, PA 15213; 412-624-8465; hooksm@pitt.edu

\section{ABSTRACT}

Understanding how feedforward inhibition regulates movement requires knowing how cortical and thalamic projections connect to inhibitory interneurons in primary motor cortex (M1). We quantified excitatory synaptic input from sensory cortex and thalamus onto two main classes of M1 inhibitory interneurons across all cortical layers: parvalbumin (PV) expressing fast-spiking cells and somatostatin (SOM) expressing low-threshold-spiking cells. Each projection innervated M1 interneurons with a unique laminar profile. While pyramidal neurons were excited by these cortical and thalamic inputs in the same layers, different interneuron types were excited in a distinct, complementary manner, suggesting feedforward inhibition from different inputs proceeds selectively via distinct circuits. Specifically, somatosensory cortex (S1) inputs primarily targeted PV+ neurons in upper layers (L2/3) but SOM+ neurons in middle layers (L5). Somatosensory thalamus (PO) inputs primarily targeted PV+ neurons in middle layers (L5). Our results show that long-range excitatory inputs target inhibitory neurons in a cell type-specific manner which contrasts with input to neighboring pyramidal cells. In contrast to feedforward inhibition providing generic inhibitory tone in cortex, circuits are selectively organized to recruit inhibition matched to incoming excitatory circuits.

(Word count: 179) 


\section{INTRODUCTION}

Motor cortex (M1) integrates long-range input from sensory cortex (S1) and thalamus to control behaviors that respond to the animal's current sensory environment. How different excitatory inputs recruit excitatory neurons has been addressed, but how differences in feedforward inhibition might shape $\mathrm{M} 1$ response properties and duration has received less attention. M1 neurons exhibit sensory activity (Ferezou et al., 2007; Hatsopoulos and Suminski, 2011; Huber et al., 2012; Murray and Keller, 2011) and are involved in active sensation (Hill et al., 2011) and sensorimotor learning (Asanuma, 1981). Input from S1 (Hoffer et al., 2003) excites pyramidal neurons primarily in upper layers of M1 (Kaneko et al., 1994a; Mao et al., 2011). Somatosensory input to M1 also originates from higher-order sensory thalamus, including the posterior nucleus (PO; Deschenes et al., 1998; Harris et al., 2019; Ohno et al., 2012) These two classes of input (S1 and PO) both most strongly excited pyramidal neurons of L2/3 and L5A (Hooks, 2017; Hooks et al., 2013; Mao et al., 2011). L2/3 and L5A neurons provide disynaptic descending input to output neurons in L5B that may regulate movement (Anderson et al., 2010; Kaneko et al., 1994b; Kiritani et al., 2012; Weiler et al., 2008). neurons. GABAergic cortical interneurons play an important role in regulating the flow of excitation in M1, but there is a great diversity of these cell types (Petilla Interneuron Nomenclature et al., 2008). Their specific connectivity and how it contributes to cortical function is unknown. Three major classes include parvalbumin-expressing $(\mathrm{PV}+)$, somatostatinexpressing $(\mathrm{SOM}+)$, and 5-HT3a receptor-expressing $(5 \mathrm{HT} 3 \mathrm{aR}+)$ interneurons (Lee et al., 2010). Of these, PV+ and SOM+ cells are found across the thickness of M1 from L2 to L6 (Fig. 1; Lee et al., 2010). PV+ interneurons include fast spiking interneurons which target perisomatic regions of pyramidal neurons and $\mathrm{SOM}+$ interneurons which include low threshold spiking interneurons. A subset of these SOM+ interneurons target apical dendrites of pyramidal cells. Differences in subcellular targeting suggests different roles in regulating cortical output. Thus, understanding how these are activated by distinct excitatory inputs is an important step in understanding how feedforward inhibition is recruited by different cortical afferents.

To test whether specific M1 afferents excite M1 interneurons in a layer- and cell typespecific pattern, we used channelrhodopsin-2 (ChR2)-assisted circuit mapping (Cruikshank et al., 2010; Petreanu et al., 2007; Petreanu et al., 2009) to quantify synaptic input strength as a function of laminar depth and specific interneuron type across layers in M1. S1 targeted interneurons differently across cell types: among PV+ neurons, L2/3 cells were most strongly excited, while for SOM+ neurons, input arrived strongly in middle layers (L5). In comparison to 
bioRxiv preprint doi: https://doi.org/10.1101/2021.07.08.451716; this version posted July 9, 2021. The copyright holder for this preprint (which was not certified by peer review) is the author/funder, who has granted bioRxiv a license to display the preprint in perpetuity. It is made available under aCC-BY 4.0 International license. layers (L5). In contrast to the strong preference of corticothalamic input for PV+ interneurons in layer 4 of sensory cortex (Cruikshank et al., 2007; Cruikshank et al., 2010), thalamic input to $\mathrm{SOM}+$ neurons was not weaker than to $\mathrm{PV}+$ neurons. Because $\mathrm{S} 1$ and $\mathrm{PO}$ targeted excitatory neurons in similar layers (Hooks et al., 2015; Hooks et al., 2013), the complementary pattern of

a

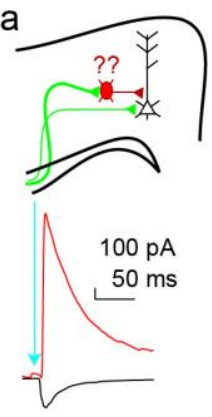

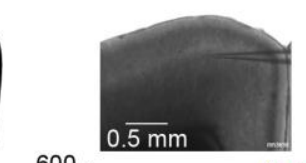

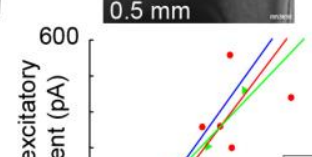

b

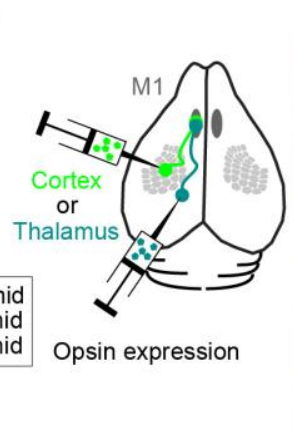

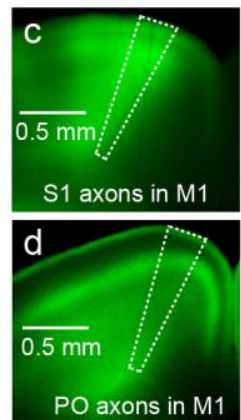

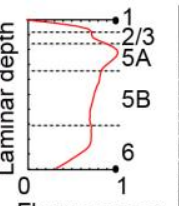

Fluorescence

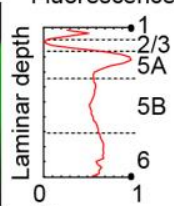

Fluorescence

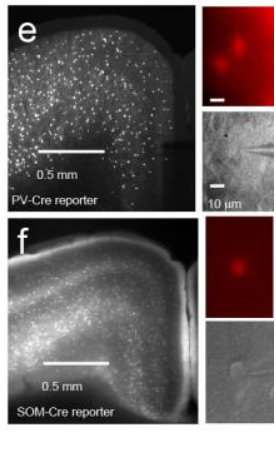

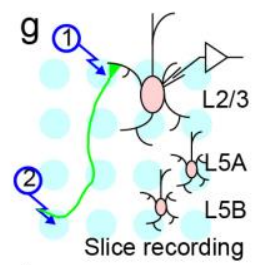

h Laser site

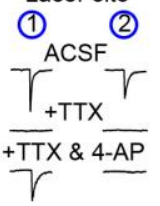
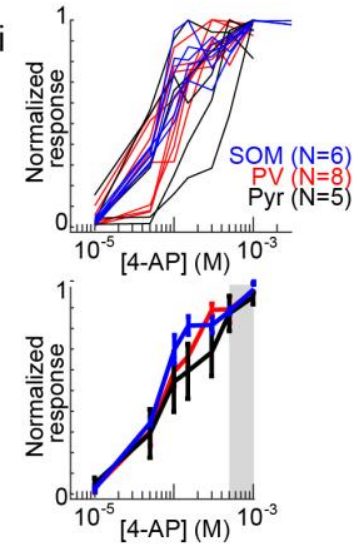
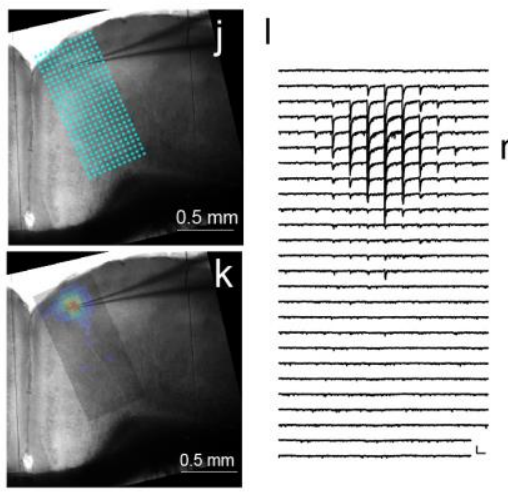

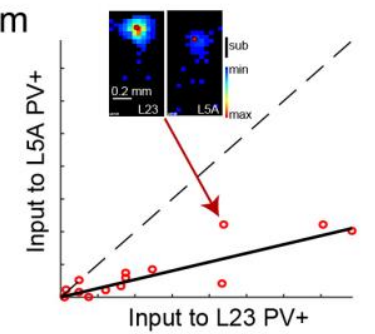

Figure 1 | Mapping long-range excitatory inputs to mouse motor cortex interneurons

(a) How do interneuron populations carry feedforward inhibition in M1? Whole-cell recordings at $-70 \mathrm{mV}$ (black) and $0 \mathrm{mV}$ (red) show optically induced feedforward inhibition from S1 inputs to M1 (left). EPSC and IPSC amplitude are roughly proportional (right) across all cortical layers. (b) AAV vectors expressing optogenetic activators (ChR2 or Chronos) were stereotaxically injected into cortex ( $\mathrm{S} 1$, light green) or thalamus (PO, dark green). (c) mVenus+ axons from S1 arborize across layers of M1 in a columnar projection (left, 4x image). Fluorescence is highest in L5A (right, brightness quantified across cortical layers). (d) mVenus+ axons from PO arborize across layers of M1 in two main layers (left). Fluorescence is highest in L1 and L5A (right). (e) PV-Cre mice were crossed to a tdTomato reporter (Ai14), labeling interneurons across M1 in L2-6. Postsynaptic interneurons were recorded across all layers for comparison. Inset (60x, fluorescence image, top, and corresponding brightfield image, bottom) shows patch pipette on tdTomato+ neuron in PV-Cre x Ai14 mouse. (f) SOM-Cre mice were crossed to a tdTomato reporter (Ai14), labeling interneurons across $M 1$ in L2-6. L1 signal represents tdTomato+ axons from SOM+ neurons. Postsynaptic interneurons were recorded across all layers for comparison. (g) Targeted whole cell recordings in brainslice were made from interneurons while stimulating with a 470nm laser at different points, shown in light blue. Full grid illustrated as in (j). (h) $470 \mathrm{~nm}$ stimulation excites axons at example points 1 \& 2 , but TTX addition extinguishes responses. Addition of 4-AP permits local release at points where opsin+ axons contact interneuron dendrites (Point 1 ) but not distant points along the axon (Point 2). (i) Similar concentrations of 4-AP are required to restore synaptic responses regardless of postsynaptic neuron type (Pyr = pyramidal neuron). Individual cells plotted at left; average and SE plotted at right. Grey box represents [4-AP] used for mapping. (j) Typical input mapping experiments sampled a $12 \times 26$ point grid with points spaced at $50 \mathrm{~mm}$ and aligned to the pia. Brightfield image $(4 \mathrm{x})$ shows recording pipette in M1. (k) Heatmap shows stronger responses (red) near the soma of the recorded neuron. (I) Example traces for a L2/3 PV+ neuron show location of input. $(\mathrm{m})$ Inset shows two maps to compare input between $L 2 / 3$ and L5A PV+ interneurons. The summed synaptic input across the map is compared for each cell pair recorded in the same slice. Arrow indicates a point from one L2/3-L5A pair. Dashed line represents $y=x$ (similar input to both layers); solid line represents the geometric mean of the L2/3 and L5A input strength, presented as a line of equivalent slope. 


\section{RESULTS}

\section{Mapping long-range excitatory inputs from sensory cortex and thalamus to M1}

Incoming cortical and thalamic excitation to M1 directly excites pyramidal neurons (Hooks et al., 2013; Mao et al., 2011) and evokes disynaptic feedforward inhibition, even in brain slice (Fig. 1a). We recorded from pyramidal neurons in the whisker region of M1 in brain slice while exciting ChR2+ axons from S1 at $-70 \mathrm{mV}$ and $+0 \mathrm{mV}$ and quantified EPSC and IPSC amplitude, respectively. Across M1 layers, excitation is matched to inhibition, with similar ratios across layers. It is likely that the GABAergic neurons carrying the disynaptic inhibition reside mainly in the same layer as the pyramidal neuron soma (Katzel et al., 2011). The interneurons carrying this feedforward inhibition are unknown, but are presumed to include PV+ basket cells.

To quantify how specific populations of interneurons are targeted by incoming cortical and thalamic afferents, we labeled axons from S1 or PO with ChR2-mVenus. These axons arborized in distinct layers of cortex (Fig. 1c-d). We selected two populations of Cre-driver mice (PV-Cre and SOM-Cre) crossed to a tdTomato reporter (Ai14; Madisen et al., 2010) in order to label genetically defined interneurons across the thickness of M1. We then targeted whole-cell recordings to tdTomato+ interneurons in M1 brain slices in the field of mVenus+ axon terminals. We used a $470 \mathrm{~nm}$ light to excite ChR2+ axon terminals while recording in TTX, CPP, and 4-AP (sCRACM; Petreanu et al., 2007; Petreanu et al., 2009). TTX prohibited action potentials, ensuring inputs measured were monosynaptic. 4-AP slowed axon repolarization, allowing the ChR2-evoked EPSC to occur (Fig. 1g-h). Because we were concerned that axons targeting different cell types might vary in their responsiveness to 4-AP, we confirmed that addition of 4AP in the presence of TTX restored synaptic release with similar effectiveness. In mapping experiments, a single interneuron was recorded while stimulating the brain slice with a $470 \mathrm{~nm}$ laser. A 12x26 grid (Fig 1j) was used for stimulation, resulting in evoked EPSCs (Fig. 1I) that differed in amplitude depending on the location of the laser stimulus. A heat map showing the size of the response was then plotted on top of the cell location in the slice (Fig. 1k). We compared each pair of interneurons in different layers recorded in the same slice, summing the EPSC amplitude across the map. This measure of input strength was used to compare EPSC amplitude across layers (Fig. $1 \mathrm{~m}$ ). Thus, our comparisons of input strength (Fig. 2-5) required pairwise comparison of neurons recorded in the same slice to normalize for ChR2 expression between animals, and nonparametric statistics were used to avoid assumptions about the

107 distribution of connection strength. 


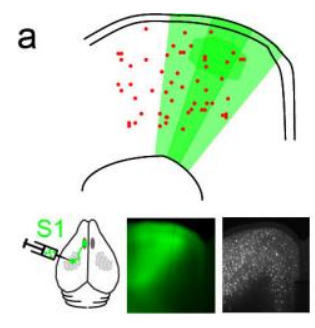

d

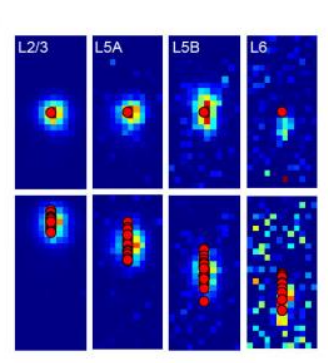

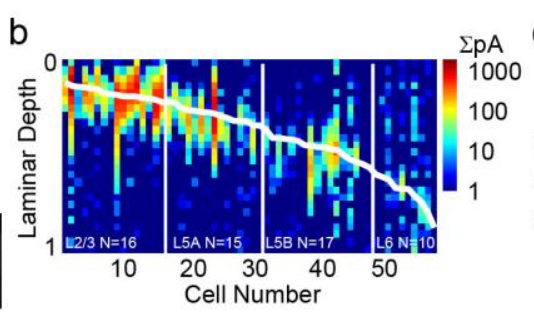

e

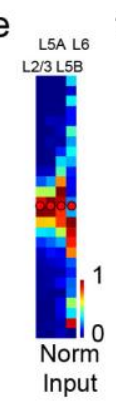

f

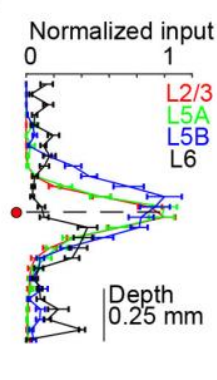

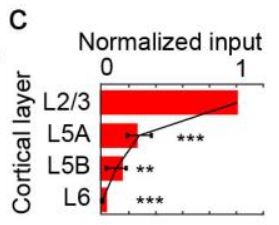
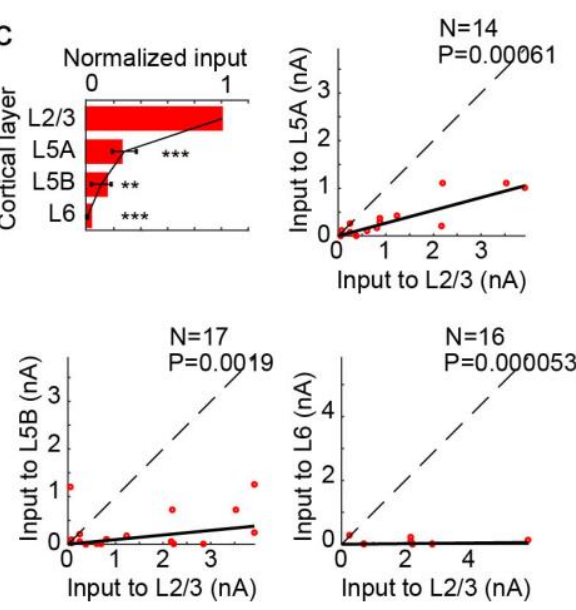

Figure 2 | S1 input to M1 targets upper layer PV+ neurons

(a) Cartoon showing PV+ neurons (red) in GFP+ axons from S1. (b) S1 input to M1 PV+ neurons across layers (N=58 total). Each cell is represented as a single column (vector), with the rows of the input mapped summed and aligned to the pia. Diagonal white line represents the soma location for that cell. Vertical white lines represent layer divisions. Synaptic strength (summed, in $\mathrm{nA}$ ) for each location in depth are represented by the heatmap (scale at right). Laminar depth is normalized to $0=$ pia, $1=$ white matter. (c) Strength of synaptic input. The bar represents the geometric means of the amplitude ratio, normalized to the layer receiving the strongest input (L23). The overlaid graph shows the mean ratio and SD (based on 10000 replicate bootstrap). Adjacent to the summary, three graphs for comparison of input strength across neurons in different cortical layers. Each point represents input to a pair of neurons in the same slice (circle for each neuron). Dashed line represents unity. N, Number of pairs; $\mathrm{p}$ value, Wilcoxon signed rank test. (d) Maps of synaptic input location in the dendritic arbor for PV+ neurons in each layer. Top row, normalized soma-centered map (maps registered to soma center across cells). Bottom row, normalized pia-aligned maps. Normalized maps are noisy when input is weak. (e,f) Input location summarized for all four layers. Normalized mean input maps were averaged into a vector (e) and aligned to the soma (triangle). These were graphed with mean and SD (f), showing input relative to the soma in $50 \mu \mathrm{m}$ bins. Dashed line indicates soma depth. ${ }^{*}=p<0.05,{ }^{* *}=p<0.01$, and ${ }^{* \star *}=p<0.001$.

\section{S1 targeting of PV+ and SOM+ interneurons in M1}

First, we recorded from tdTomato+ PV+ interneurons in the whisker region of M1 in PVCre x Ai14 mice. S1 inputs to M1 mainly targeted PV+ interneurons closest to the pia. These layer 2/3 PV+ neurons received more than twice as much input than any other layer. All PV+ neurons $(\mathrm{N}=58)$ in all layers were plotted for comparison (Fig. 2b), with each map reduced to a column vector by summing the input map across rows. Most responsive cells and the strongest responding cells were found in $L 2 / 3$. A pairwise comparison of $L 2 / 3$ with other $P V+$ neurons recording in the same slice showed that $L 5 A$ neurons received only about $30 \%$ the input of $L 2 / 3$ PV+ neurons $(P<0.001)$. Similarly, input to L5B and L6 was even weaker (Fig. 2c). This input was mostly centered around the interneuron soma, as shown in maps where the grid is aligned to the soma (Fig. 2d-e).

We repeated this experiment for $\mathrm{S} 1$ inputs to SOM+ interneurons, using SOM-Cre $\times$ Ai14 mice (Fig. 3). As in PV-Cre mice, SOM-Cre labeled interneurons across the cortical thickness, allowing a fair comparison of input strength across layers. Here, S1 inputs most strongly targeted L5A interneurons. L2/3 SOM+ neurons only received about 30\% the input of L5A SOM+ cells (Fig. 3c, p<0.05). L5B was weaker as well $(\sim 40 \%)$, though not significantly so 


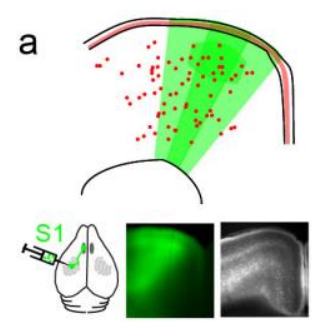

d

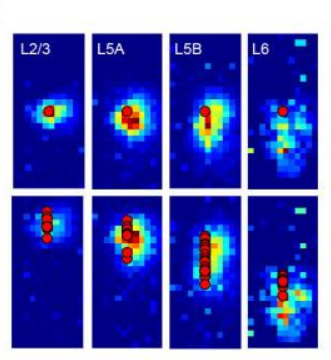

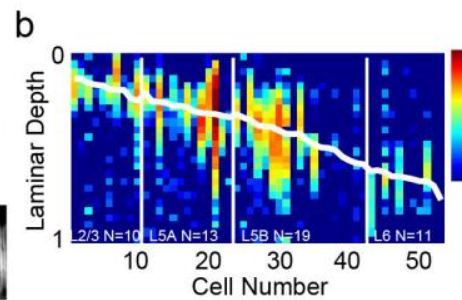

e L5ALE $f$

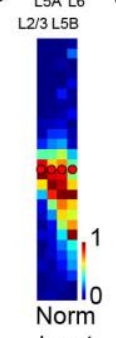

Input
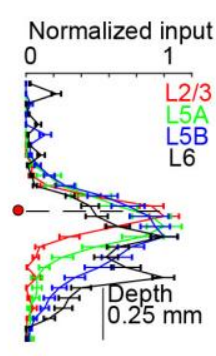
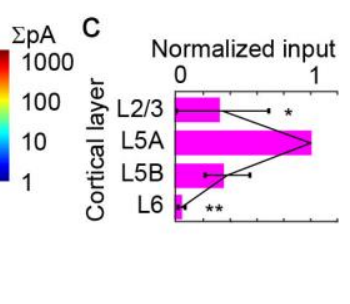
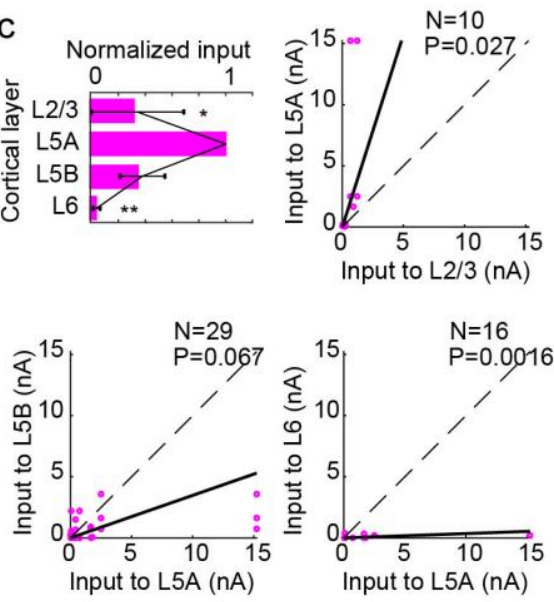

Figure 3 | $\mathbf{S} 1$ input to $M 1$ targets middle layer SOM+ neurons

(a) Cartoon showing SOM+ neurons (red) in GFP+ axons from S1. (b) S1 input to SOM+ neurons across layers (N=53 total). Each cell is represented as a single column (vector), with the rows of the input mapped summed and aligned to the pia. Diagonal white line represents the soma location for that cell. Vertical white lines represent layer divisions. Synaptic strength (summed, in $\mathrm{nA}$ ) for each location in depth are represented by the heatmap (scale at right). Laminar depth is normalized to $0=$ pia, $1=$ white matter. (c) Strength of synaptic input. The bar represents the geometric means of the amplitude ratio, normalized to the layer receiving the strongest input (L5A). The overlaid graph shows the mean ratio and SD (based on 10000 replicate bootstrap). Adjacent to the summary, three graphs for comparison of input strength across neurons in different cortical layers. Each point represents input to a pair of neurons in the same slice (circle for each neuron). Dashed line represents unity. $\mathrm{N}$, Number of pairs; $\mathrm{p}$ value, Wilcoxon signed rank test. (d) Maps of synaptic input location in the dendritic arbor for SOM+ neurons in each layer. Top row, normalized somacentered map (maps registered to soma center across cells). Bottom row, normalized pia-aligned maps. Normalized maps are noisy when input is weak. (e,f) Input location summarized for all four layers. Normalized mean input maps were averaged into a vector (e) and aligned to the soma (triangle). These were graphed with mean and SD (f), showing input relative to the soma in $50 \mu \mathrm{m}$ bins. Dashed line indicates soma depth.

$(\mathrm{p}=0.067)$. L6 SOM+ neurons received the least input $(\mathrm{p}<0.01)$. As with $\mathrm{PV}+$ neurons, $\mathrm{S} 1$ input was generally perisomatic, especially for L2/3 SOM+ cells. However, the center of mass of the input was shifted below the soma towards white matter for SOM+ cells in L5A, 5B, and 6 (Fig. different interneuron types.

\section{PO targeting of PV+ and SOM+ interneurons in M1}

Next, we repeated these experiments while labeling thalamic inputs from PO nucleus of thalamus, a higher order somatosensory nucleus whose input to M1 pyramidal neurons had been characterized (Hooks et al., 2015; Hooks et al., 2013). Thalamic injections were determined in individual animals to be in PO and not anterior motor nuclei by the presence of axonal fluorescence in L1 and L5A. The absence of L5B fluorescence in these animals confirmed that our injections did not target motor thalamic nuclei, such as the anterior components of the ventroanterior-ventrolateral complex, which had previously been shown to arborize in three distinct bands (L1, L5A, and L5B) instead of two (Hooks et al., 2013). Targeting recordings to tdTomato+ $\mathrm{PV}+$ interneurons within the arborization of $\mathrm{PO}$ axons, we found that, in contrast to $S 1$ inputs, neurons in $L 5 A$ received the strongest synaptic input (Fig. 4c). 


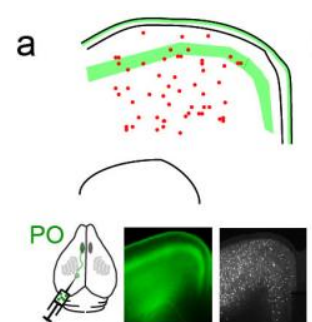

d

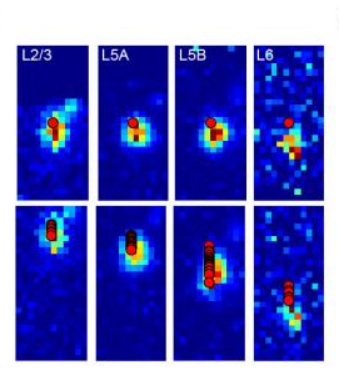

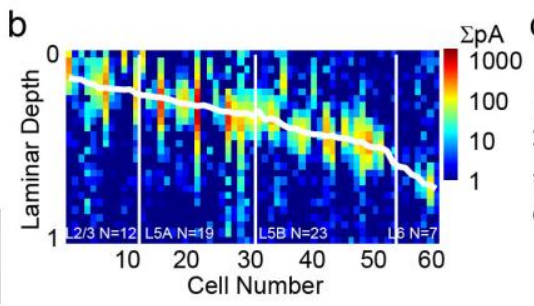

e ${ }_{\text {LSALE }} f$

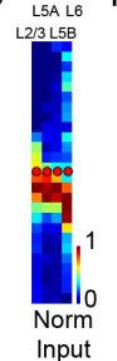

Normalized input

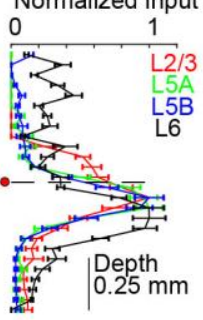

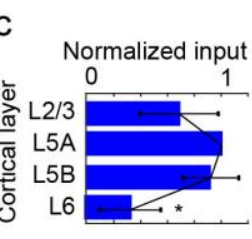
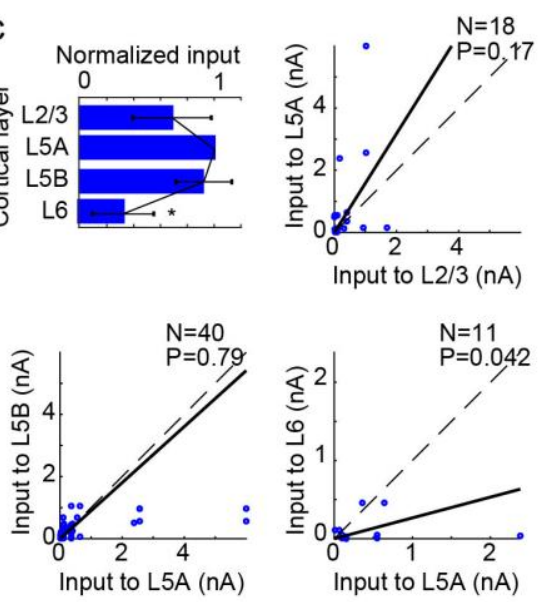

Figure 4 | PO input to M1 PV+ neurons is targets upper and middle layers

(a) Cartoon showing PV+ neurons (red) in GFP+ axons from PO. (b) PO input to PV+ neurons across layers (N=61 total). Each cell is represented as a single column (vector), with the rows of the input mapped summed and aligned to the pia. Diagonal white line represents the soma location for that cell. Vertical white lines represent layer divisions. Synaptic strength (summed, in nA) for each location in depth are represented by the heatmap (scale at right). Laminar depth is normalized to $0=$ pia, $1=$ white matter. (c) Strength of synaptic input. The bar represents the geometric means of the amplitude ratio, normalized to the layer receiving the strongest input (L5A). The overlaid graph shows the mean ratio and SD (based on 10000 replicate bootstrap). Adjacent to the summary, three graphs for comparison of input strength across neurons in different cortical layers. Each point represents input to a pair of neurons in the same slice (circle for each neuron). Dashed line represents unity. N, Number of pairs; $\mathrm{p}$ value, Wilcoxon signed rank test. (d) Maps of synaptic input location in the dendritic arbor for PV+ neurons in each layer. Top row, normalized soma-centered map (maps registered to soma center across cells). Bottom row, normalized pia-aligned maps. Normalized maps are noisy when input is weak. (e,f) Input location summarized for all four layers. Normalized mean input maps were averaged into a vector (e) and aligned to the soma (triangle). These were graphed with mean and SD (f), showing input relative to the soma in $50 \mu \mathrm{m}$ bins. Dashed line indicates soma depth.

However, input to PV+ cells in the top three layers of motor cortex (L2/3, L5A, and L5B) was generally strong, without significant differences. Only synaptic input to L6 was weaker $(\mathrm{P}<0.05)$. This input was not centered on the soma in the dendritic arbor, but instead was generally targeted to the deep (white matter) side of proximal dendrites (Fig. 4d-f). This was the case for all four layers measured (L2/3, L5A, L5B, and L6). Thalamic injections were targeted to PO and not anterior motor nuclei, which could be discerned in individual animals by the presence of axonal fluorescence in L1 and L5A. The absence of L5B fluorescence in these animals suggested that our injections did not target motor thalamic nuclei, such as the anterior components of the ventroanterior-ventrolateral complex, which had previously been shown to excite L5B pyramidal neurons (Hooks et al., 2013).

$\mathrm{PO}$ inputs to $\mathrm{SOM}+$ interneurons were more difficult to characterize. We used the same approach (Fig. 5) to quantify synaptic input. However, the pattern of input to SOM+ was noisy and did not match any previous pattern observed for the other GABAergic cells (Fig 2-4) nor pyramidal neurons previously recorded (Hooks et al., 2013; Mao et al., 2011). Instead, we found that L5B was the most strongly excited layer (Fig. 5c), with significantly reduced input in L5A $(p<0.001)$. Comparisons to L2/3 and L6 were not significant and showed quite different patterns across different cells, with some pairs favoring L2/3 or L6 and others favoring L5B. The 

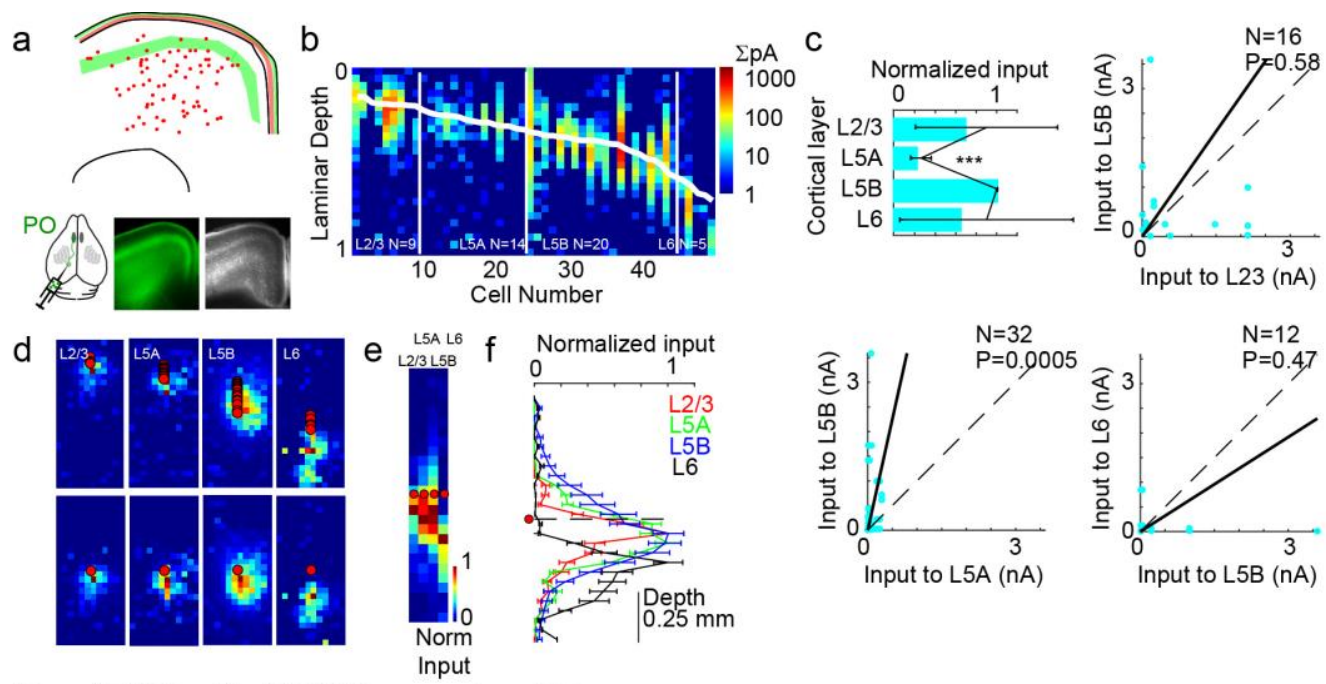

Figure 5 | PO input to M1 SOM+ neurons is variable

(a) Cartoon showing SOM+ neurons (red) in GFP+ axons from PO. (b) PO input to SOM+ neurons across layers ( $\mathrm{N}=48$ total). Each cell is represented as a single column (vector), with the rows of the input mapped summed and aligned to the pia. Diagonal white line represents the soma location for that cell. Vertical white lines represent layer divisions. Synaptic strength (summed, in $\mathrm{nA}$ ) for each location in depth are represented by the heatmap (scale at right). Laminar depth is normalized to $0=$ pia, $1=$ white matter. (c) Strength of synaptic input. The bar represents the geometric means of the amplitude ratio, normalized to the layer receiving the strongest input (L5B). The overlaid graph shows the mean ratio and SD (based on 10000 replicate bootstrap). Adjacent to the summary, three graphs for comparison of input strength across neurons in different cortical layers. Each point represents input to a pair of neurons in the same slice (circle for each neuron). Dashed line represents unity. N, Number of pairs; $\mathrm{p}$ value,

Wilcoxon signed rank test. Plots show substantial heterogeneity. (d) Maps of synaptic input location in the dendritic arbor for SOM+ neurons in each layer. Top row, normalized soma-centered map (maps registered to soma center across cells). Bottom row, normalized pia-aligned maps. Normalized maps are noisy when input is weak. (e,f) Input location summarized for all four layers. Normalized mean input maps were averaged into a vector (e) and aligned to the soma (red circle). These were graphed with mean and SD (f), showing input relative to the soma in $50 \mu \mathrm{m}$ bins. Dashed line indicates soma depth.

subcellular localization of input was also heterogeneous, with some SOM+ neurons in L5B and L6 receiving input deep to the soma.

\section{Complementary patterns of input to specific interneuron types}

By comparing synaptic strength across layers and normalizing excitability to the strongest input layer for multiple cell types, we were able to compare the pattern of input for the same pathway to multiple cell types. We plotted the normalized input strength graph on the same axes for S1 inputs or PO inputs to PV+ and SOM+ cells, as well as the same data grouped by input to PV+ or SOM+ cells (Fig. 6a-d). This enabled a direct comparison of $\mathrm{S} 1$ and $\mathrm{PO}$ input to $\mathrm{PV}+$ and $\mathrm{SOM}+$ neurons (Fig. 6a). Here, the same presynaptic afferents preferred interneurons of different types in different layers. Considering the same data organized by postsynaptic target, cortical inputs from S1 to PV+ cells strongly preferred the uppermost (L2/3) PV+ cells, while the functional targeting of $P O$ inputs was biased toward deeper layers (L5A and L5B; Fig. 6c). Input to SOM+ neurons showed a similarly complementary pattern, with input from S1 axons most strongly exciting L5A, which is the layer

175 least strongly excited by PO (Fig. 6d). Thus, the same type of interneuron is targeted in a

176 different manner by the cortical and thalamic inputs. For comparison, we plotted the input 

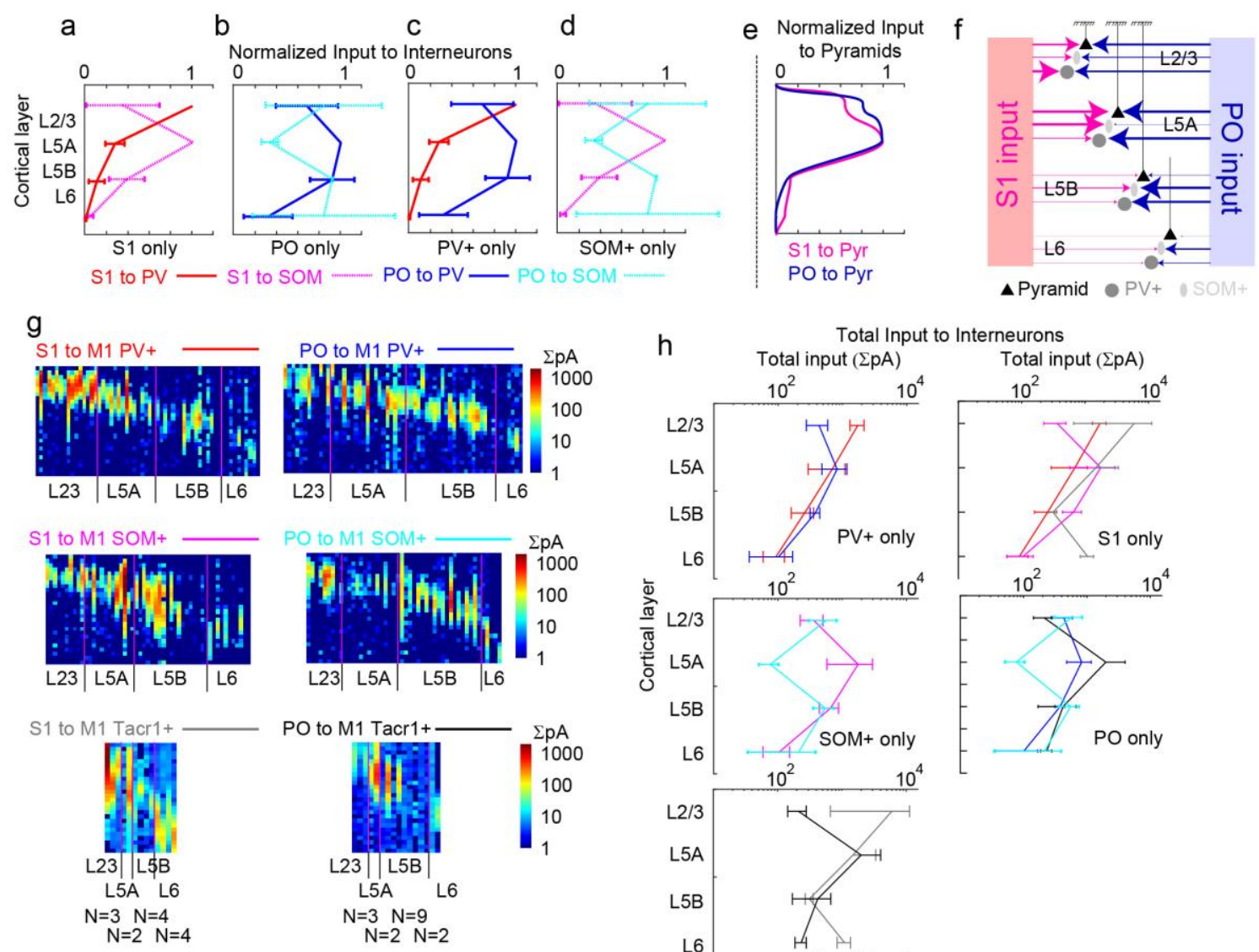

g

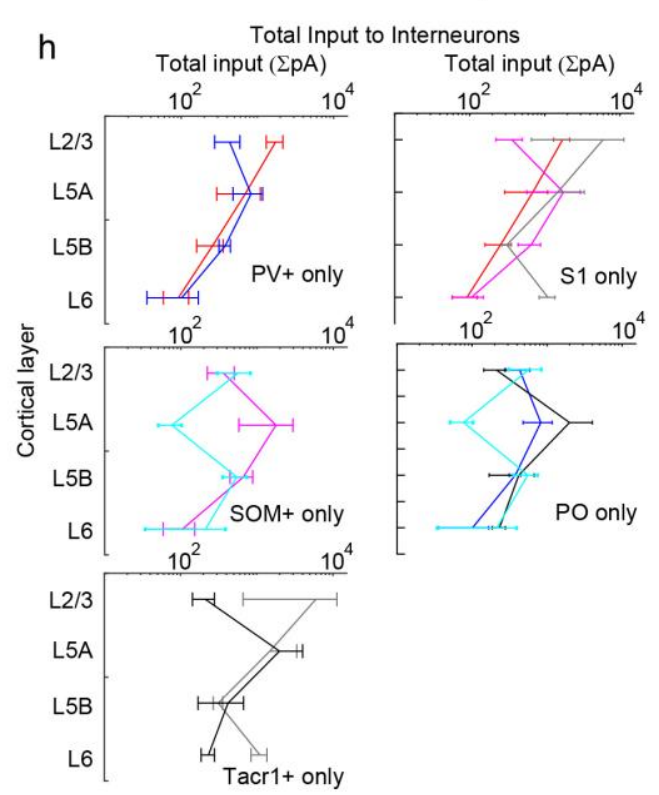

Figure 6 | Complementary patterns of input to specific interneuron types

(a-d) Summary graphs comparing the distribution of excitatory input to $\mathrm{PV}+$ and $\mathrm{SOM}+$ neurons. These plots compare normalized input from (a) $\mathrm{S} 1$ only or (b) PO only to PV+ and SOM+ neurons. (c-d) compare input to PV+ only (c) or SOM+ only interneurons. (e) The pattern of normalized input strength to pyramidal neurons (Pyr) from S1 and PO, after Mao et al (2011) and Hooks et al. (2013). (f) Cartoon summarizing input maps. (g) Absolute input strength for $\mathrm{S} 1$ and $\mathrm{PO}$ inputs, plotted as input vectors on a log scale. Top row, PV+ neurons; middle row, SOM+ neurons, bottom row, Tacr1+ neurons (a subset of SOM+ neurons). Left column, S1 inputs. Right column, PO inputs. Data presented as in Figs 2-5. For Tacr1+ neurons, $\mathrm{N}=3 / 2 / 4 / 4$ for $\mathrm{S} 1$ input to $\mathrm{L} 2 / 3, \mathrm{~L} 5 \mathrm{~A}, \mathrm{~L} 5 \mathrm{~B}$, and $\mathrm{L} 6 ; \mathrm{N}=3 / 2 / 9 / 2$ for $\mathrm{PO}$ input. (h) Absolute input strength averaged by layer and plotted on a log scale for comparison between $\mathrm{S} 1$ and PO inputs to different interneuron types (left column) or input from S1 or PO (right column).

strength to excitatory neurons (Hooks, 2017). This pattern shows that S1 and PO generally target pyramidal neurons in the same layer (Fig. 6e). We summarize all the synaptic strength experiments schematically in Fig. 6f, where arrow thicknesses to specific cell types indicate celltype specific connectivity.

Because these inputs are plotted as normalized input strength, we wondered whether the absolute strength of input to $\mathrm{PV}+$ and $\mathrm{SOM}+$ differed across pathways. We compared input across pathways and cell types by plotting the absolute input strength for all cells on the same scale, as other investigators have done (Kinnischtzke et al., 2014). This was done by summing the EPSC amplitudes across the points of the input map (as in Fig. 11). Cell vector plots were presented as before (Fig 6g) using the same logarithmic scale for all plots to improve presentation of more frequent recordings with weaker inputs. In general, excitatory input from $\mathrm{S} 1$ and $\mathrm{PO}$ to either interneuron type ( $\mathrm{PV}+$ or SOM+, top and middle row) was of similar magnitude. Averaging input by layer confirmed that monosynaptic input was of comparable 
strength (Fig. 6h). The overall pattern is similar to our normalized data. The distribution of $\mathrm{S} 1$ and $\mathrm{PO}$ input to $\mathrm{PV}+$ (Fig. 6h top) is nearly overlapping, with the exception of input to L2/3 PV+ neurons. Input to SOM+ neurons is of similar strength from S1 and PO except for L5A, where the absence of thalamic input is pronounced. Corticocortical input is similar or stronger to SOM+ neurons than PV+ neurons at each laminar depth except L2/3 (Fig. 6h, top right).

Thalamocortical input is stronger to L5A PV+ neurons than to SOM+ cells. Outside of this layer, however, input strength to $\mathrm{SOM}+$ cells is equal to $\mathrm{PV}+$ neurons (Fig. 6h, middle right).

Because SOM+ neurons comprise a diverse range of neurons, including Martinotti and non-Martinotti subsets (Tremblay et al., 2016), we were curious whether the input pattern to the SOM+ neurons labeled in our SOM-Cre x Ai14 strategy would be able to be replicated in a more targeted subset of cells (Huang et al., 2016; Tasic et al., 2018). We labeled the Tacr1+ subset of interneurons using a NK1R-CreER mouse crossed to a tdTomato reporter. Like SOM+ and $\mathrm{PV}+$ crosses, this resulted in labeled neurons across the cortical thickness. We repeated our input mapping experiments in these mice and presented the data in a similar format (Fig. $6 \mathrm{~g}$ ). In contrast to all SOM+ and PV+ neurons, Tacr1+ cells responded well to S1 inputs across multiple layers. Of note, the strongest responses were in in $L 2 / 3$ and $L 6$, with weaker input to $L 5$ (Fig. 6f-h). The input from $\mathrm{PO}$ also did not correspond to the SOM+ pattern, with strongly responding neurons in L5A and L5B.

\section{Short term plasticity of long-range excitation to PV+ and SOM+ neurons}

Our measurements of synaptic strength were based on monosynaptic input under recording conditions (TTX, CPP, 4-AP) which monitor synaptic responses to the first stimulus.

These measurements might not reflect the relative contribution of the two interneuronal populations to a train of stimuli, which might be more physiological. Since PV+ and SOM+ neurons show differences in short term plasticity of local excitatory inputs (Beierlein et al., 2003), we were interested to test whether later synaptic inputs in a train might alter which interneuron population was more likely to carry feedforward inhibition.

To measure short term synaptic plasticity, we shifted to expressing a kinetically rapid channelrhodopsin, Chronos (Klapoetke et al., 2014). We stimulated Chronos-GFP+ afferents at 10, 20, and $40 \mathrm{~Hz}$ using a $470 \mathrm{~nm}$ LED (Fig. 7). Sweeps were averaged and data normalized to the amplitude of the first pulse. For S1 inputs to PV+ neurons, the synaptic inputs were depressing for all frequencies, and most strongly depressing for higher frequencies (Fig. 7a, right). Similarly, $\mathrm{PO}$ inputs to $\mathrm{PV}+$ neurons were also depressing, though this pattern was quantitatively different. The degree of depression was greater for PO inputs than for $\mathrm{S} 1$, and the 

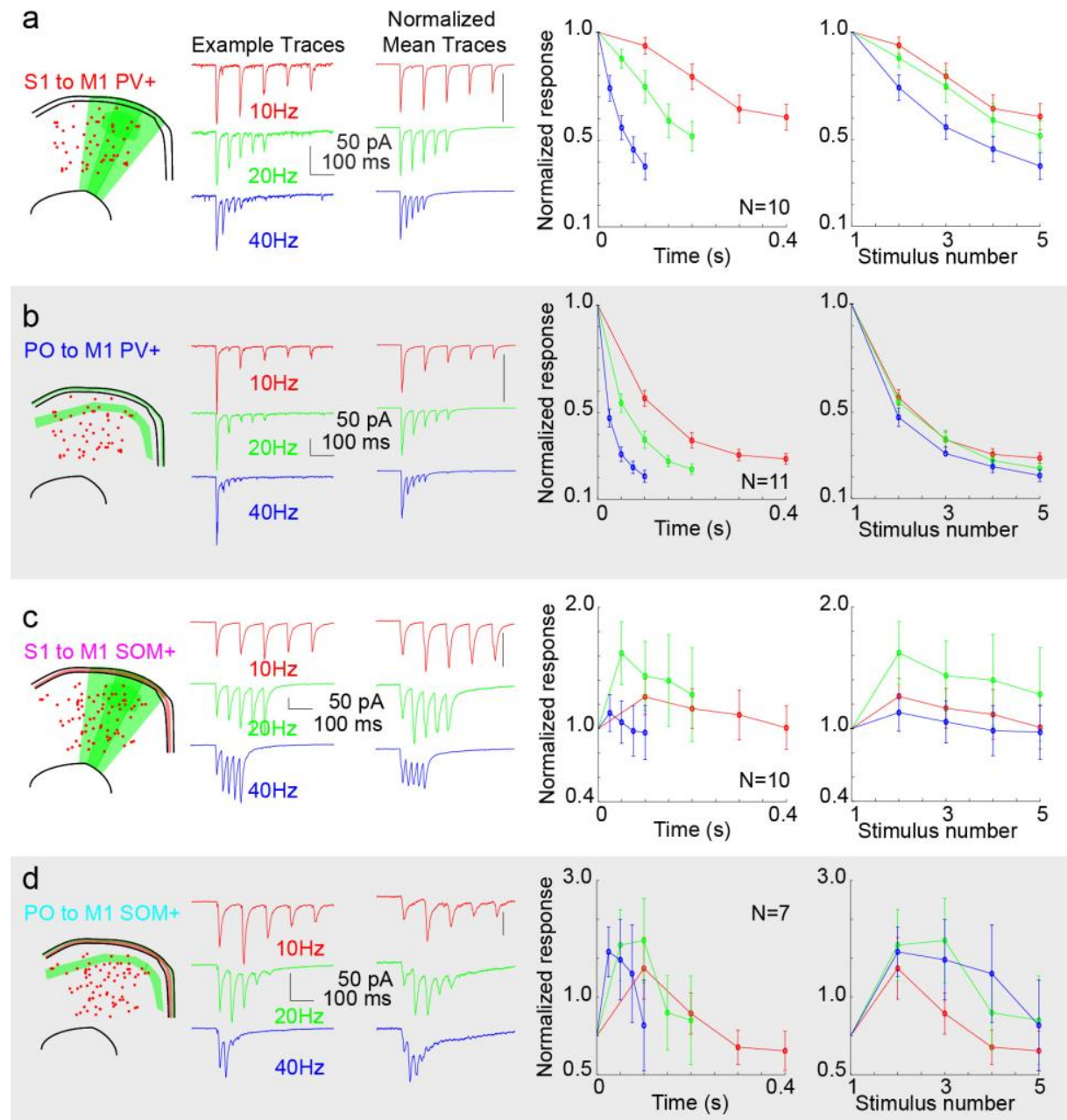

Figure 7 | Short-term plasticity at long range inputs to interneurons of mouse motor cortex

(a) Short term plasticity of S1 input to PV+ neurons. Left, example traces showing PV+ neuron response to S1 stimulation at $10 \mathrm{~Hz}$ (red), $20 \mathrm{~Hz}$ (green), and $40 \mathrm{~Hz}$ (blue) stimulation of Chronos-GFP+ S1 axons. Scale, $50 \mathrm{pA}$ and $100 \mathrm{~ms}$. Middle, average normalized traces for the same stimulus frequencies. Scale bar $=1.0$. Right, summary graphs showing normalized responses for $10 \mathrm{~Hz}, 20 \mathrm{~Hz}$, and $40 \mathrm{~Hz}$. Graphs are plotted based on time of the pulses (right) or the stimulus number (far right). (b) Short term plasticity of PO input to PV+ neurons, plotted as in (a). (c) Short term plasticity of S1 input to SOM+ neurons, plotted as in (a). (d) Short term plasticity of PO input to SOM+ neurons, plotted as in (a).

depression was comparable for the same stimulus at 10, 20, and $40 \mathrm{~Hz}$, suggesting recovery from short term depression is slower in PO than in $\mathrm{S} 1$ afferents. Repeating these experiments in pyramidal neurons showed qualitatively similar depressing inputs from S1 and PO (Supp. Fig. $1)$.

In contrast to these results in $\mathrm{PV}+$ neurons, SOM+ cells showed some degree of

230 Similarly, PO input was also more facilitating at 20 and $40 \mathrm{~Hz}$. These experiments were

231 performed using the same viral vector and recording conditions for both mouse lines, was not due exclusively to an artifact of measuring short term plasticity with optical methods 
(Jackman et al., 2014). We do not currently have tools to distinguish whether the long-range axons that innervate $\mathrm{PV}+$ and $\mathrm{SOM}+$ neurons are branches from the same presynaptic neurons of whether these are two independent pathways. It is thus possible that the same axon might have different release properties depending on its cellular target (Beierlein et al., 2003).

\section{Anatomical tracing of long-range inputs to $\mathrm{PV}+$ and $\mathrm{SOM}+$ neurons}

Our functional circuit mapping suggested some quantitative differences in the strength with which two of the major cortical and thalamic inputs connected to specific subsets of M1 interneurons. Differences in short-term plasticity further suggested means by which the strength of connections to $\mathrm{PV}+$ and $\mathrm{SOM}+$ neurons may differ. Conventional retrograde and anterograde tracers can identify presynaptic cortical (Dum and Strick, 2002; Hoffer et al., 2003; Reep et al., 1990; Rouiller et al., 1993) and thalamic (Deschenes et al., 1998; Kuramoto et al., 2009; Kuramoto et al., 2015; Ohno et al., 2012) inputs to M1 (Hooks et al., 2013; Oh et al., 2014; Zingg et al., 2014), but cannot differentiate between cell types. We thus used genetically identified interneurons as starter cells for monosynaptic retrograde tracing with rabies virus (Callaway and Luo, 2015; Wickersham et al., 2007). This enabled us to qualitatively and quantitatively compare anatomical approaches to our functional circuit mapping data, as well as to explore other brain areas providing input to M1.

Using PV-Cre or SOM-Cre mice, we infected Cre+ interneurons with AAV-DIO-TVAEGFP-B19G to make potential starter neurons that would express both TVA receptor (to become infected with EnvA pseudotyped rabies virus) as well as the rabies coat protein B19G (Fig. 8a-d). The starter cells in these injections spanned cortical laminae. After two weeks to express the receptor, EnvA-pseudotyped CVS-N2c G-deleted rabies expressing tdTomato (Reardon et al., 2016) was injected in forelimb motor cortex (fM1, N=5 for PV+, N=2 for SOM+ mice; see Table 3). Fixed brain were sectioned, immunoamplified, and imaged. Reconstructed whole brain image stacks were aligned to the Allen CCF V3.0 (Kuan et al., 2015; Oh et al., 2014). tdTomato+ neurons were detected and quantified based on their position in the reference atlas (Fig. 8e). fM1 was used to be able to later compare the data to results from the Brain Initiative Cell Census Network (BICCN) studies (Muñoz-Castaneda, 2020). This approach was further limited since it was not possible to restrict starter $\mathrm{PV}+$ or $\mathrm{SOM}+$ neurons to a single cortical layer. The number of presynaptic neurons was normalized as a fraction of the total number of tdTomato+ neurons detected (Fig. 8j). Overall, this pattern was relatively similar for inputs to $\mathrm{PV}+$ and $\mathrm{SOM}+$ neurons. Most presynaptic inputs were found in cortex, in particular frontal and motor areas on the dorsal surface of cortex, as well as somatosensory cortex, which 

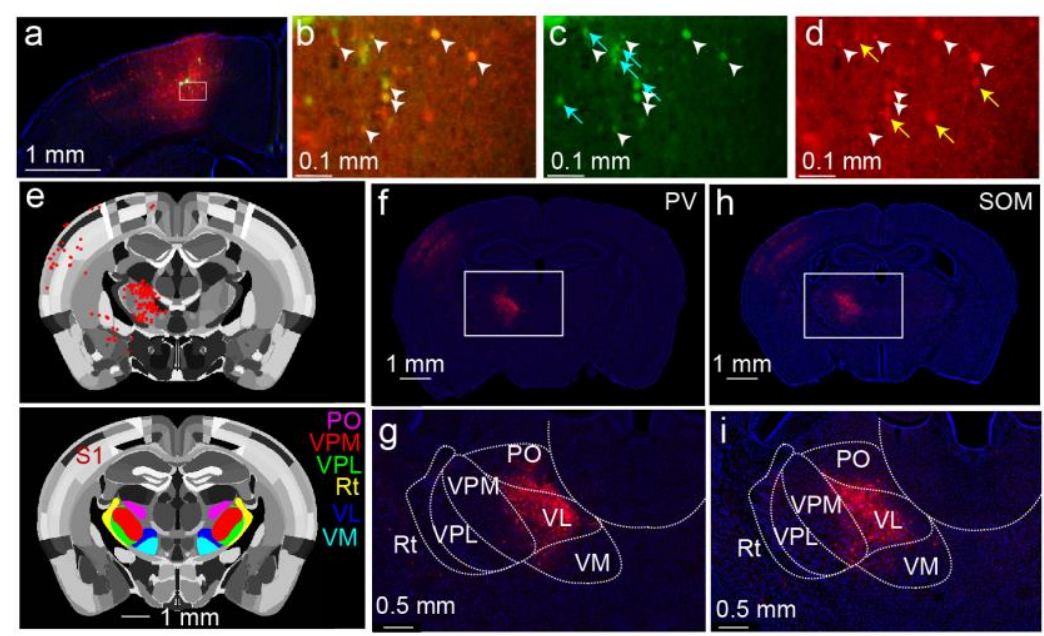

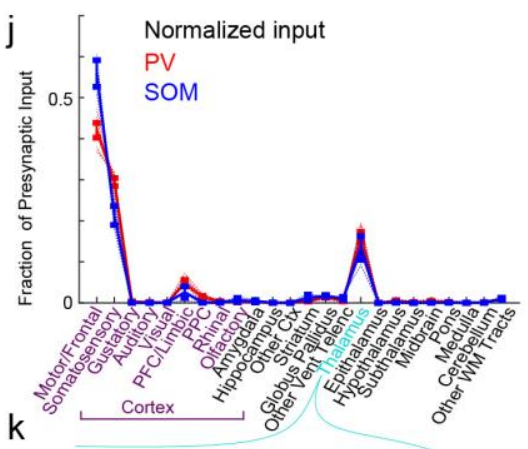

$\mathrm{k}$

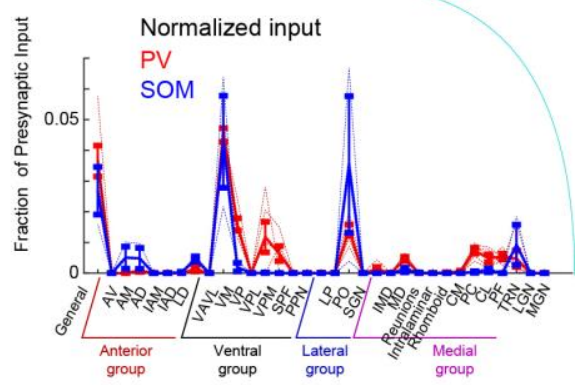

Figure 8 | Monosynaptic Retrograde Tracing of Inputs to Motor Cortex PV+ and SOM+ Neurons

(a-d) Injection site in M1 of a SOM-Cre mouse injected with AAV-DIO-TVA-RG-EGFP and EnvA pseudotyped N2c RABV-tdTomato. (a) Low magnification image. Blue, Neurotrace; Green, TVA+ neurons (putative starter cells); Red, tdTomato+ neurons (putative presynaptic neurons). (b) Merged image of green (c) and red (d) channels, showing yellow starter cells (arrowheads) and singly labeled cells (blue arrows in c and yellow arrows in d). (e) Coronal section showing the Allen CCF 3.0 and locations of S1 and motor and somatosensory thalamic nuclei (PO, posterior nuc. of thalamus; S1, primary somatosensory cortex; VL, ventrolateral nuc.; VM, ventromedial nuc.; VPL, ventroposterolateral nuc.; VPM, ventroposteromedial nuc.). The positions of retrogradely labeled presynaptic neurons are marked with a red dot (top image) in an example SOM-Cre mouse injected in M1. Presynaptic neurons within $\pm 0.1 \mathrm{~mm}$ of the plane are shown. The bottom image (more posterior plane) is labeled to indicate cortical and thalamic areas. (f-g) Example section showing retrograde label in S1 and thalamus from M1 starter cells in a PV-Cre mouse. Note S1 label and intense thalamic label. (h-i) Example section showing retrograde label in S1 and thalamus from M1 starter cells in a SOM-Cre mouse. Note S1 label and intense thalamic label. (j) Quantification of presynaptic neuron location. Whole brain image stacks were aligned to the Allen CCF V3.0.

Presynaptic neuronal somata (red cells) were detected and coordinates in CCF assigned to brain regions. Fraction of the total presynaptic neurons population plotted for all motor cortex injections in PV-Cre $(\mathrm{N}=5$, red) and SOM-Cre $(\mathrm{N}=2$, blue) mice. Dotted lines represent individual cases; solid line represents mean. (k) Quantification of thalamic inputs for all motor and frontal injections in $\mathrm{PV}-\mathrm{Cre}(\mathrm{N}=5$, red) and $\mathrm{SOM}-\mathrm{Cre}(\mathrm{N}=2$, blue) mice. Data plotted as in (j), subdivided into distinct thalamic nuclei.

is strongly reciprocally connected to mouse M1 (Hooks, 2017; Mao et al., 2011). Limbic areas, especially orbital areas, provided some input, which more substantial in projects to M2 for both SOM+ and PV+ interneurons (Supp. Fig. 2a,c). About 20\% of inputs for both interneuron classes was localized to thalamus (Fig. 8j). Comparisons of thalamic input between PV+ and $\mathrm{SOM}+$, however, were not perfectly overlapping. Instead, some nuclei were more strongly retrogradely labeled from SOM+ neurons, including $\mathrm{PO}$. This is consistent with our circuit mapping data (Fig. 6h). By expanding this study to PV+ and SOM+ starter neurons in different locations across cortex, we were able to examine thalamocortical input for cell type-specific targeting. Quantifying thalamic input by specific thalamic nucleus suggested that patterns differed both across cortical areas, with different thalamic nuclei labeled by injections in vS1,

$278 \mathrm{fM} 1$, and M2 (Supp. Fig 2b,d). Furthermore, the strength of labeling might differ between

279 interneuron types (Supp. Fig. 2 f,g,h). 


\section{DISCUSSION}

At the circuit level, anatomical models for cortical connectivity based on overlap of axons and dendrites (Braitenberg and Schuz, 1998) propose that the potential for functional connectivity is proportional to this overlap. A range of excitatory and inhibitory neurons exist in cortex (Tasic et al., 2018; Tremblay et al., 2016), each with potentially different rules for establishing connectivity as well as plasticity. Thus, it is possible that each cortical cell type will have its own specific connectivity pattern. Here we show that cortical and thalamic long-range inputs to M1 excite two types of inhibitory interneurons in different and complementary ways. S1 inputs most strongly targeted L2/3 PV+ neurons, but SOM+ neurons in middle layers (L5). Further, while S1 and PO both targeted pyramidal neurons in L2/3 and L5A with similar connection strength, these pathways targeted $\mathrm{PV}+$ neurons in a different fashion. These results indicate that cortical and thalamic afferents form highly specific connections in neocortex. Thus, the recruitment of feedforward inhibition may not be simply a blanket of inhibition, but instead is divided into specific feedforward circuits.

What is the functional relevance of this? A circuit organization where incoming afferents activate a selected subset of interneurons suggests that certain inputs seek to activate some cortical networks while silencing others. For M1, this might mean activating the appropriate response to incoming sensory stimuli, while inhibiting others. This would help in the selection of one action over others. Notably, while both S1 and PO pathways excite L5B more weakly than other layers (Hooks et al., 2013; Mao et al., 2011), PO excited both PV+ and SOM+ interneurons in this layer, which might aid in terminating signals associated with inappropriate motor responses. Similarly, short-term plasticity differences in facilitation and depression may shape the timing and duration of inhibition. Thus, M1 computations performed on feedforward excitatory inputs from to S1 and PO input may be shaped by the relative amounts of facilitating and depressing inhibition recruited.

\section{Specific circuits for feedforward inhibition}

Motor cortex integrates a wide range of input from frontal cortical areas (Dum and Strick, 2002; Reep et al., 1990; Rouiller et al., 1993), primary and secondary sensory cortex (Chakraibarti and Alloway, 2006; Hoffer et al., 2003; Kaneko et al., 1994a; Smith and Alloway, 2013; Suter and Shepherd, 2015), retrosplenial cortex (Yamawaki et al., 2016), and different thalamic nuclei (Deschenes et al., 1998; Kuramoto et al., 2009; Ohno et al., 2012; Strick and Sterling, 1974). These inputs activate M1 during voluntary and sensory guided movement, with each targeting excitatory neurons in M1 in a layer-specific manner (Hooks et al., 2013). Different 
M1 neurons can represent either sensory input or motor behavior such as whisking, licking, or lever pressing (Harrison and Murphy, 2014; Huber et al., 2012; Masamizu et al., 2014; Tanaka et al., 2018), suggesting that individual neurons are parts of different networks, receiving input of different strength from cortical inputs. Here we tested whether this pattern is the same for input to interneurons, finding instead that the laminar pattern of excitation also varies with the postsynaptic cell type targeted.

Each excitatory pathway evokes to some degree feedforward inhibition (Isaacson and Scanziani, 2011). This disynaptic inhibition might serve in part to terminate continuing excitation from the same inputs. Once recruited, local circuit interneurons connect to large numbers of nearby pyramidal neurons (Bock et al., 2011; Fino and Yuste, 2011; Packer and Yuste, 2011). If synaptic weights are strong, this might provide an effective suppression of cortical activity (Fino et al., 2013), though the effectiveness of these connections could be reweighted by activitydependent (Xue et al., 2014) or experience-dependent (Chen et al., 2015) plasticity mechanisms. Thus, it is of interest to understand whether the recruitment of feedforward inhibition proceeds through distinct interneurons (of either the same or different type) for inputs recruiting motor cortex. Our data indicates that there is space for selectivity in inhibitory circuitry.

Some specific examples of differences stand out in our quantitative maps. Our data suggests that the incoming sensory information from S1 excites $\mathrm{PV}+$ neurons in L2/3 but SOM+ neurons in L5. Since these same afferents from S1 also strongly target pyramidal neurons in L2/3 and L5A (Mao et al., 2011), it suggests that, in part, the feedforward inhibition recruited to L2/3 pyramids and L5A pyramids can proceed through different neural populations, as cortical interneurons generally, though not exclusively, inhibit pyramidal neurons in the same layer (Katzel et al., 2011). Since SOM+ neurons may also inhibit PV+ cells, helping to disinhibit cortex (Pfeffer et al., 2013), it is possible that S1 input can have differential inputs on the excitability of L2/3 and L5A pyramids, despite similarity in monosynaptic excitation to these targets. This would suggest that differences in cell types might play a role in determining the efficacy of feedforward input and the rules of long term plasticity in these two target layers. The pattern of thalamic input to $\mathrm{PV}+$ interneurons also differed from S1 input. This suggests that where cortical and thalamic afferents target the same excitatory network, to the extent S1 and PO target partially non-overlapping PV+ populations, feedforward inhibition recruited by one pathway would not be as strongly depressed by the recruitment of PV+ inhibition by the other pathway arriving at a similar time.

Of note, PO provided fairly strong input to both $\mathrm{PV}+$ and SOM+ neurons in L5B (Fig. 6fh). This feedforward inhibition would potentially inhibit L5B pyramidal neurons, which are only 
weakly excited by S1 and PO L5A (Hooks et al., 2013; Mao et al., 2011). We infer the net effect would be to reduce activity in most L5B pyramidal neurons, though it is unknown if there is specific targeting to certain pyramidal neuron subtypes. L5B contains two main classes of

352 output neurons, IntraTelecephalic type cells projecting to both cortex and striatum, as well as

353 Pyramidal Tract type cells which mainly target thalamus, brainstem, and spinal cord (Hooks et

354 al., 2018; Shepherd, 2013). Thus, a range of cortical output channels might be suppressed. The

355 function of inhibiting neurons outside the normal targets of excitation of these pathways is

356 unknown, but by reducing overall activity of M1 output neurons might serve to set a higher

357 threshold of activation in the context of incoming sensory information. By reducing background

358 activation, this would increase the signal to noise ratio of outgoing motor signals.

\section{Subcellular organization of input to interneurons}

Our circuit mapping approach gives some insight into the subcellular localization of synaptic input in the dendritic arbor of postsynaptic neurons. This insight is constrained by methodological limitations of patch clamp which limit our ability to study distal dendrites. Interneurons, however, are generally more electronically compact than pyramidal neurons. There is some organization of the input around the $\mathrm{PV}+$ and $\mathrm{SOM}+$ soma, suggesting some mechanisms for afferent targeting exist and may differ between interneuron subtypes. S1 input to $\mathrm{PV}+$ neurons is evenly distributed perisomatically, while these same afferents tend to target dendrites $\sim 50-150 \mu \mathrm{m}$ below the soma of SOM+cells. Similarly, PO input to PV+ cells is also shifted deep to the soma center by $\sim 50-150 \mu \mathrm{m}$. This offset is interesting in contrast to thalamic inputs to pyramidal neurons (Petreanu et al., 2009). In these cells, PO input targets the apical

371 dendrites, and especially the L1 arbors of L3 pyramidal neurons but is soma-centered (and quite

372 strong) for L5A pyramidal neurons. This is consistent with each cell type directing input from a

373 defined presynaptic pathway to some extent within its arbor.

\section{Organization of long range input to SOM+ interneurons differs across cortical areas} received weaker input than neighboring pyramidal neurons and $\mathrm{PV}+$ neurons (Bruno and

378 Simons, 2002; Cruikshank et al., 2007; Cruikshank et al., 2010). This thalamocortical preference

379 for PV+ over SOM+ neurons in somatosensory cortex extends to L2/3 (Naskar et al., 2021).

380 Thalamic input to SOM+ does exist in sensory areas, though it may be developmentally downregulated at ages younger than those studied here (Tuncdemir et al., 2016). 
However, we were surprised to find many layers where SOM+ cells received comparable inputs to PV+ cells as measured by monosynaptic input mapping (Porter et al., 2001). Although comparisons are challenging due to limitations of normalizing input strength across different injections in different animals (Petreanu et al., 2009), the mean thalamic input strength to SOM+ interneurons was marginally higher than that to PV+ neurons except for L5A (Fig. 6h). However, there is considerable variability between individual cells (Fig. $6 \mathrm{~g}$ ). Similarly, corticocortical input from S1 was higher to SOM+ neurons in L5A and L5B (Fig. 6h top). This collectively suggests that, it is not a hard-and-fast rule that thalamic input will be selective for $\mathrm{PV}+$ neurons across all areas and layers. Consistent with this, retrograde viral tracing data suggests that large numbers of neurons in $\mathrm{S} 1$ and $\mathrm{PO}$ can be labeled projecting to both $\mathrm{PV}+$ and $\mathrm{SOM}+$ neurons in $\mathrm{M} 1$ (Fig. 8). The molecular mechanisms underlying this difference in target specificity are unknown and perhaps the postsynaptic targets help instruct this developmentally. Though individual PO axons are quite widespread in cortex, and project to multiple cortical areas in some reconstructions (Ohno et al., 2012), data suggests that cortico-thalamo-cortical loops in M1 and S1 are segregated (Guo et al., 2020). Thus, the same individual axon might not need to make more common connections to SOM+ targets in $\mathrm{M} 1$ while demonstrating different target selectivity in S1.

In addition to monosynaptic input strength, it is also worth considering that input from S1 and PO may not occur in isolation but as part of trains of action potentials, especially should thalamus fire in burst mode (Sherman, 2001; Steriade et al., 1993). In the case of PO, short term depression of input to $\mathrm{PV}+$ neurons was the strongest short term plasticity we observed (Fig. 7). In contrast, these inputs were facilitating to $\mathrm{SOM}+$ neurons. This suggests that as rapidly as the second or third pulse in a train, the recruitment of feedforward inhibition could shift from PV+ to SOM+ neurons (Tan et al., 2008). The difference in short term facilitation and depression to $\mathrm{SOM}+$ and $\mathrm{PV}+$ neurons respectively is not as large for $\mathrm{S} 1$ afferents as for $\mathrm{PO}$ inputs, though the general pattern is the same. This effect is qualified by differences in the intrinsic excitability of the particular cells involved, as well as their own depression onto

409 pyramidal neuron targets. Inhibition from SOM+ neurons is hypothesized to be facilitating, in 410 contrast to the depression observed in fast spiking neurons (Beierlein et al., 2003). If this is the 411 case in M1, then this would serve to further shift the balance of disynaptic feedforward inhibition 412 from $\mathrm{PV}+$ to $\mathrm{SOM}+$ neurons, giving SOM+ neurons the potential to play a role in regulating 413 excitability and plasticity (Chen et al., 2015).

$414 \quad$ Our anatomical mapping experiments with monosynaptic rabies were also consistent 415 with distinct rules for thalamic input to interneuron types depending on the cortical area 
targeted. Coarsely, our data show that cortical areas differ only in their connectivity to thalamus and cortex, since these are the only areas strongly projecting to any cortical region we studied. Cortical connectivity is specific to each region, of course: M2 gets input from limbic area while M1 and S1 do not (Supp. Fig. 2a and 2c). But corticocortical maps were generally similar whether input was traced from PV+ or SOM+ cells (Supp. Fig. 2e, $2 g$, and 2i). However, thalamic differences appeared more substantial, as expected since each area has its own

422 principal input. Input from VA/VL nuclei were most strongly connected to fM1 for both SOM and

423 PV with some label from M2 as well. MD and VM were most strongly connected to M2 for both

424 interneuron types as well; the strength of MD label provided a fingerprint in our quantification for

$425 \mathrm{M} 2$ injections. VPM was specific for S1. PO provides input across M2, fM1, and S1, consistent with the range of axonal arborizations of these cells (Ohno et al., 2012). But there is room for some target specificity: retrograde tracing from vS1, as expected (Cruikshank et al., 2007;

428 Cruikshank et al., 2010), labeled VPM and PO input more strongly from PV+ than SOM+ neurons (Supp. Fig. 2j). But, for fM1 and M2 this preference was reversed, consistent with our functional circuit mapping data.

\section{Different SOM+ subtypes have different connectivity} variability within this dataset, especially for thalamic inputs (Fig. 5). In our assessment of monosynaptic input strength and short-term plasticity, $\mathrm{PV}+$ neurons behaved in a much more homogeneous manner. These are the largest GABAergic neuron population ( 40\%), consisting of mostly perisomatic-targeting basket cells, though this group also includes axon-targeting chandelier cells (Tremblay et al., 2016). In contrast, SOM+ neurons ( 30\%) include distal dendrite targeting Martinotti-type neurons, evident in the L1 axonal fluorescence in Fig 1f, as well as non-Martinotti types. Thus, SOM+ neurons include at least two (and likely more) molecular subtypes (Ma et al., 2006; Tasic et al., 2018; Zeisel et al., 2015), which might account for the variability in the data. One SOM+ subset of interneurons expresses Tacr1, as well as NPY and nNOS (Kubota et al., 2011), though these may be subdivided into two subsets based on nNOS expression level (Dittrich et al., 2012). Tacr1+ cells, like both PV+ and SOM+ neurons

446 Notably, this was not the case for VIP+ neurons, which are much more numerous in only upper 447 layers (L2/3), as well as other non-VIP expressing $5 \mathrm{HT} 3 \mathrm{aR}+$ interneurons which are similarly 448 biased to L1 and L2/3 (Lee et al., 2010). 
When synaptic strength was mapped, presented a pattern of input strength quite in contrast to that of the SOM-Cre x Ai14 mice (Fig. 6f). In particular, S1 input had been distributed across L2/3, L5A, and L5B to SOM+ neurons, but most strongly to L5A. In contrast, this subset had a quite distinct patter, with substantial L2/3 and L6 input. PO input was noisy to $\mathrm{SOM}+$ neurons in general. This emphasized the likelihood that each molecular subtype of interneuron had some mechanism to govern synaptic strength.

Overall, these results point towards circuit mechanisms by which different pathways of incoming excitation may excite specific subcircuits, include specific networks for inhibition in primary motor cortex. Having some means by which recruitment of feedforward inhibition from each pathways does not generally silence all M1 circuits is perhaps necessary for control of movement when $\mathrm{M} 1$ is receiving competing inputs from different brain regions.

\section{MATERIALS AND METHODS}

Stereotactic injections. Animal protocols were approved by Institutional Animal Care and Use Committees at University of Pittsburgh. Mice of either sex were used. Experimental procedures were similar to previous studies (Hooks et al., 2013). Cre driver mouse lines (PV-Cre, SOM-Cre, and NK1R-CreER) were used in conjunction with the Isl-tdTomato reporter line, Ai14, to label specific interneuron populations (see Table 1). Tacr1 experiments using NK1R-CreER, required tamoxifen injections to drive recombination. Animals were anesthetized using isoflurane and placed in a custom stereotactic apparatus. Juvenile mice at postnatal $(P)$ day P12-P22 were injected with AAV expressing excitatory opsins. AAVs included CAG-ChR2-mVenus and hSynChronos-GFP. AAV-CaMKIla-hChR2(H134R)-EYFP was used in Tacr1 mapping experiments

472 (see Table 2 for details and serotypes). Injections were made with glass pipettes (Drummond)

473 using a custom-made positive displacement system (Narashige). Stereotactic coordinates are

474 listed in Table 3. A pair of injections (50-100 nL; 500 and $800 \mu \mathrm{m}$ depth from pia) was made in

475 the cortex. One location $(50-100 \mathrm{~nL})$ was injected into thalamic targets.

476 Electrophysiology and photostimulation. Brain slices were prepared $>14$ days after viral 477 infection, in young adult mice ( P30-P60). Mice were anesthetized with isoflurane and the brain 478 was rapidly removed and placed in cooled choline-based cutting solution (in $\mathrm{mM}: 110$ choline 479 chloride, 3.1 sodium pyruvate, 11.6 sodium ascorbate, $25 \mathrm{NaHCO}_{3}, 25 \mathrm{D}$-glucose, $7 \mathrm{MgCl}_{2}, 2.5$ $\left.480 \mathrm{KCl}, 1.25 \mathrm{NaH}_{2} \mathrm{PO}_{4}, 0.5 \mathrm{CaCl}_{2}\right)$. Off-coronal sections $(300 \mu \mathrm{m})$ of $\mathrm{M} 1$ were cut using a vibratome 481 (VT1200S, Leica). Additional sections were cut to confirm injection location. Slices were incubated at $37^{\circ} \mathrm{C}$ in oxygenated ACSF (in mM: $127 \mathrm{NaCl}, 25 \mathrm{NaHCO}_{3}, 25 \mathrm{D}$-glucose, $2.5 \mathrm{KCl}$, 
$\left.2 \mathrm{CaCl}_{2}, 1 \mathrm{MgCl}_{2}, 1.25 \mathrm{NaH}_{2} \mathrm{PO}_{4},\right)$ for $>30$ min and maintained at room temperature (22 $\left.{ }^{\circ} \mathrm{C}\right)$ thereafter.

Whole cell recordings were performed at $22{ }^{\circ} \mathrm{C}$ in oxygenated ACSF with borosilicate pipettes (3-6 M $\Omega$; Warner Instruments) containing potassium gluconate-based internal solution (in $\mathrm{mM}: 128$ potassium gluconate, $4 \mathrm{MgCl}_{2}, 10 \mathrm{HEPES}, 1 \mathrm{EGTA}, 4 \mathrm{Na}_{2} \mathrm{ATP}, 0.4 \mathrm{Na}_{2} \mathrm{GTP}, 10$ sodium phosphocreatine, 3 sodium L-ascorbate; $\mathrm{pH}$ 7.27; 287 mOsm). These recordings targeted neurons in whisker M1 for whiskers (vM1, coordinates in Table 3). In some experiments, biocytin was added to the intracellular solution ( $3 \mathrm{mg} / \mathrm{mL}$ biocytin or neurobiotin). For subcellular ChR2-Assisted Circuit Mapping (sCRACM) experiments (Petreanu et al., 2009), TTX (1 $\mu \mathrm{M}$, Tocris), 4-AP (0.1-0.3 mM, Sigma) and CPP (5 $\mu \mathrm{M}$, Tocris) were added to the bath. Dose-response of 4-AP concentration was confirmed in a subset of experiments comparing EPSC amplitude while 4-AP was added. Under these conditions, laser pulses (1 ms) depolarized ChR2-expressing axons in the vicinity of the laser beam and triggered the local release of glutamate. Measurements of postsynaptic currents then revealed the presence of functional synapses between ChR2-expressing axons and the recorded neuron near the photostimulus. Blocking action potentials prevented polysynaptic contributions.

Data were acquired at $10 \mathrm{kHz}$ using an Axopatch 700B (Molecular Devices, Sunnyvale, CA) and Ephus software (www.ephus.org) (Suter et al., 2010) on a custom-built laser scanning photostimulation microscope (Shepherd et al., 2003). During SCRACM mapping, neurons were held at $-70 \mathrm{mV}$. A blue laser (473 nm, CrystaLaser) was controlled via scan mirrors (Cambridge Technology). Light pulses were controlled with a shutter (4 ms open time) in series with an acousto-optic modulator (1 ms pulse, Quanta-Tech) to deliver $\sim 0.5-2 \mathrm{~mW}$ at the specimen plane through a low power objective (4x, 0.16 NA, UPlanApo; Olympus). Laser power was constant during a given day. Sweep consisted of $100 \mathrm{~ms}$ baseline and $300 \mathrm{~ms}$ following onset of the stimulus. The map grid (12x26 sites at $50 \mu \mathrm{m}$ spacing) was centered horizontally over the soma of the recorded neuron, aligned at its upper edge to the pia, and covered the entire interneuron dendritic arbor. Maps were repeated 2-4 times and averaged across trials. Stimuli for short term plasticity were delivered using $1 \mathrm{~ms}$ flashes of a $470 \mathrm{~nm}$ LED (Cairn). Sweeps were interleaved and repeated 4-6 times per stimulus frequency.

Data analysis. Data analysis was performed with custom routines written in Matlab.

Electrophysiology data were low pass filtered $(1 \mathrm{kHz})$. EPSCs were detected with a threshold of $>6 x$ standard deviation from baseline). Mean EPSC amplitude for input maps was averaged over 75 ms post-stimulus (reported in pA). For comparison between cells in the same slice, total input was computed by summing supratheshold pixels. Paired comparisons across cells use the 
nonparametric Wilcoxon signed-rank test. Mean ratio of excitation were calculated as geometric means, with the strongest input layer shown as 1.0. In these comparisons, the bar graph was overlaid with a line showing the bootstrap mean and SD (10000 replicates) resampling the paired data. To compare the axonal profile of S1 and PO axons (Fig. 1), we quantified the fluorescence as before (Hooks et al., 2013) by determining the mean fluorescence of pixels at a given laminar depth (binned in 50 bins across cortex from pia to white matter). $\mathrm{N}$ are reported in figures for number of cells. Number of mice used are: $\mathrm{N}=15$ ( $\mathrm{S} 1$ to $\mathrm{M} 1 \mathrm{PV}+$ ), N=12 (PO to M1 $\mathrm{PV}+$ ), $\mathrm{N}=10$ (S1 to $\mathrm{M} 1 \mathrm{SOM}+), \mathrm{N}=10$ (PO to M1 SOM+), N=4 (S1 to M1 Tacr1+), and N=4 (PO to $\mathrm{M} 1 \mathrm{Tacr} 1+)$.

Monosynaptic viral tracing. We used AAV injections to express DIO-TVA-EGFP-B19G in potential starter cells in cerebral cortex of PV-Cre and SOM-Cre driver mouse lines (Atasoy et al., 2008; Wickersham et al., 2007). After two weeks of expression time, we injected EnvA pseudotyped rabies (CVS-N2 ${ }^{\Delta G}$ strain) into cortex and allowed 8-9 days for transport and expression (Reardon et al., 2016). Following transcardial perfusion, whole brains were sectioned, immunoamplified for EGFP and TdTomato, and stained with NeuroTrace Blue (ThermoFisher) as a structural marker. The brain was imaged and reconstructed as previously described (Eastwood et al., 2019; Hooks et al., 2018). Original images are posted at: http://gerfenc.biolucida.net/link?=Jl1tV7 (Biolucida Cloud Viewer, free download) and http://gerfenc.biolucida.net/images/?page=images\&selectionType=collection\&selectionld=32 (web-based). Images were aligned to the Allen CCF V3.0 (Kuan et al., 2015; Oh et al., 2014) using Neurolnfo software (MBF Bioscience). Somata were detected by Neurolnfo. Their coordinates in the Allen CCF used to quantify in which structure the neuron was detected using custom Matlab software. Presynaptic labeled (tdTomato+) neurons in each structure were quantified as a fraction of total labeled neurons in that brain to normalized for differences between mice and injections. 


\section{TABLES}

545

546

\section{Table 1. Mouse lines}

\begin{tabular}{lll} 
Mouse Lines & Line \# & Reference \\
\hline PV-Cre & JAX 017320 & (Hippenmeyer et al., 2005) \\
SOM-Cre & JAX 017320 & (Taniguchi et al., 2011) \\
Ai14 & JAX 007914 & (Madisen et al., 2010) \\
NK1R-CreER & JAX 035046 & (Huang et al., 2016)
\end{tabular}

547

548

\section{Table 2. Viral reagents}

\begin{tabular}{lll} 
Virus & Serotype & Addgene \# \\
\hline CAG-ChR2-mVenus & AAV1, AAV9, and AAV10 & 20071 \\
hSyn-Chronos-GFP & AAV1, AAV5, and AAV9 & 59170 \\
CaMKIla-hChR2(H134R)-EYFP & AAV9 & 26969 \\
DIO-TVA-EGFP-B19G & AAV1 & 52473 \\
N2c- $\Delta$ G-EnvA-tdTomato & CVS-N2c rabies & n/a \\
\hline
\end{tabular}

Table 3. Injection coordinates

551 Anterior/posterior (A-P) axis reported relative to bregma (positive values anterior to bregma).

552 Medial/lateral (M-L) axis reported relative to the midline. Dorsal/ventral (D-V) axis, depth from 553 pia. Injections were made at both depths in cortex. Distances in $\mathrm{mm}$.

\begin{tabular}{llll} 
Target & A-P & M-L & D-V \\
\hline Primary whisker motor cortex (vM1) & +1.1 & 0.9 & $0.5 / 0.8$ \\
Posterior thalamus (PO) & -1.2 & 1.3 & 2.75 \\
Primary whisker somatosensory cortex (vS1) & -0.6 & 3.0 & $0.5 / 0.8$ \\
For rabies tracing & & & \\
Secondary motor cortex (M2) & +2.6 & 1.0 & $0.5 / 0.8$ \\
Forelimb motor cortex (fM1) & +0.6 & 1.5 & $0.5 / 0.8$ \\
Primary whisker somatosensory cortex (vS1) & -0.6 & 3.0 & $0.5 / 0.8$
\end{tabular}


bioRxiv preprint doi: https://doi.org/10.1101/2021.07.08.451716; this version posted July 9, 2021. The copyright holder for this preprint (which was not certified by peer review) is the author/funder, who has granted bioRxiv a license to display the preprint in perpetuity. It is made available under aCC-BY 4.0 International license.
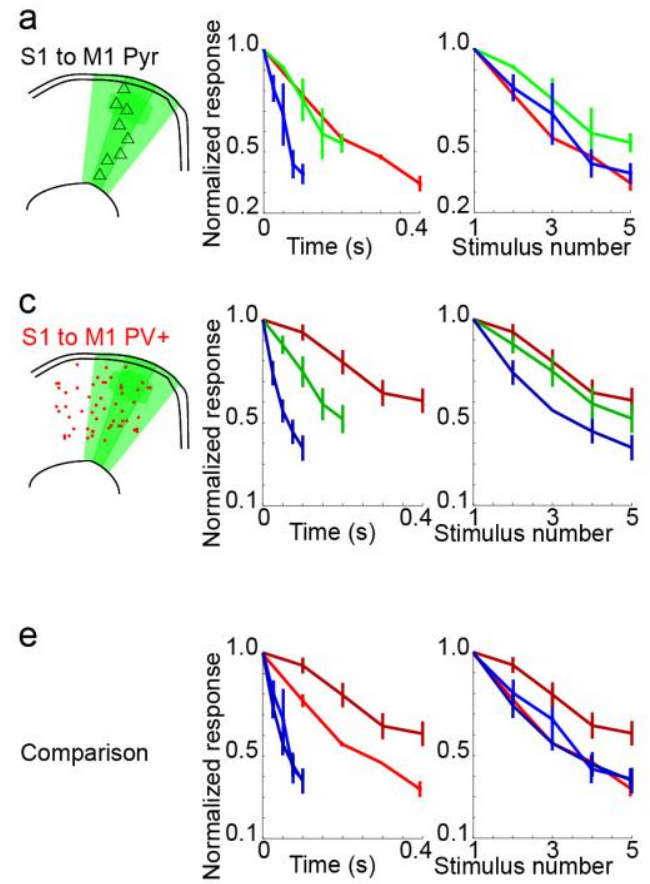

b
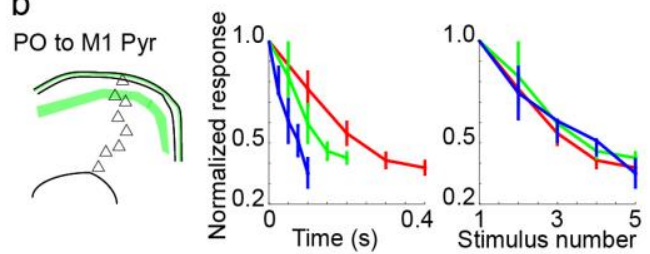

d

$\mathrm{PO}$ to $\mathrm{M} 1 \mathrm{PV}+$
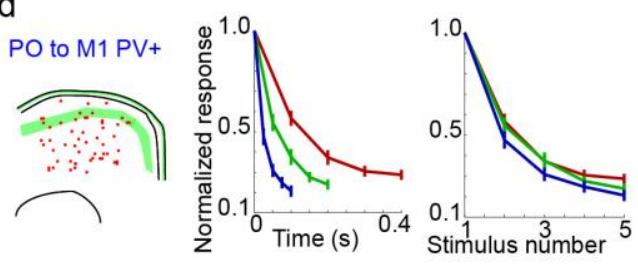

f

Comparison

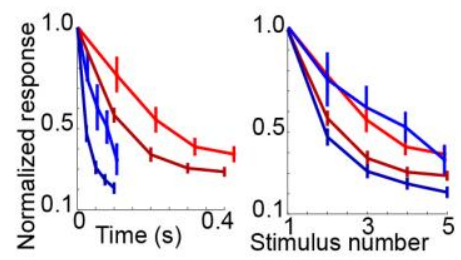

Supplemental Figure 1 | Short-term plasticity comparisons of PV+ interneurons and pyramidal neurons

(a) Short term plasticity of S1 input to pyramidal neurons. Left, normalized response to $\mathrm{S} 1$ stimulation at $10 \mathrm{~Hz}$ (red), $20 \mathrm{~Hz}$ (green), and $40 \mathrm{~Hz}$ (blue) stimulation of Chronos-GFP+. Summary graphs show responses plotted based on time of the pulses (left) or the stimulus number (right). (b) Short term plasticity of PO input to pyramidal neurons, plotted as in (a). (c,d) Short term plasticity of S1 input (c) and PO input (d) to PV+ interneurons plotted for comparison. (e,f) Comparison plot of the above data for $10 \mathrm{~Hz}$ and $40 \mathrm{~Hz}$ stimulation on the same axes. 
bioRxiv preprint doi: https://doi.org/10.1101/2021.07.08.451716; this version posted July 9, 2021. The copyright holder for this preprint (which was not certified by peer review) is the author/funder, who has granted bioRxiv a license to display the preprint in perpetuity. It is made available under aCC-BY 4.0 International license.
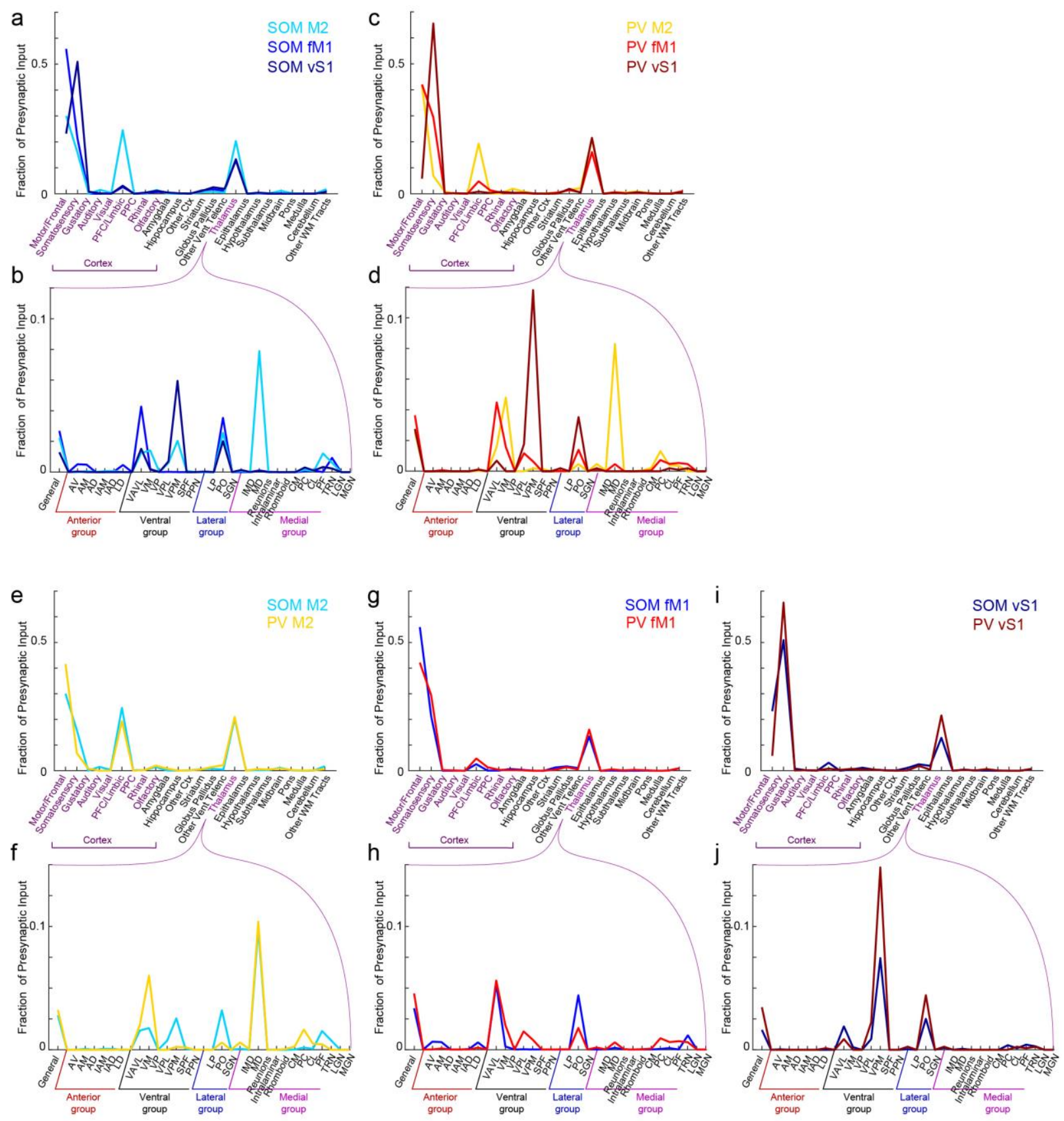

Supplemental Figure 2 | Monosynaptic Retrograde Tracing of Inputs to Cortical PV+ and SOM+ Neurons (a-b) Quantification of presynaptic neuron location for injections in SOM-Cre ( $N=2, M 2 ; N=2, f M 1 ; N=1$, vS1; cool colors) mice. Whole brain image stacks were aligned to the Allen CCF V3.0. Presynaptic neuronal somata were detected and coordinates in CCF assigned to brain regions. Mean fraction of the total presynaptic neurons population plotted as in Fig. 8. Quantification of inptus to all brain regions (a) and to thalamic nuclei (b). (c-d) Data from injections in PV-Cre ( $\mathrm{N}=2, \mathrm{M} 2 ; \mathrm{N}=5, \mathrm{fM} 1 ; \mathrm{N}=1, \mathrm{vS} 1$; hot colors) plotted as in (a-b). (e-j) Data of (a-d) reploteed to compare injections tracing input to SOM+ and PV+ neurons in the same cortical locations: M2 (e-f), fM1 (g-h), and vS1 (i-j). 


\section{ACKNOWLEDGEMENTS}

We thank Caroline Runyan, Chinfei Chen, Nuo Li, Srivatsun Sadagopan, Taehyeon Kim, Shelby Ruiz, and other members of the Hooks lab for comments and suggestions. Jay Couey performed whole cell recordings contributing to the circuit mapping and short-term plasticity experiments. This work was supported by a NARSAD Young Investigator Award (BMH), NIH NINDS R01 NS103993 (BMH), and a CDMRP PRMRP Discovery Award PR201842 (RG and $\mathrm{BMH})$. The authors declare no competing financial interests or other conflict of interest.

\section{REFERENCES}

Anderson, C.T., Sheets, P.L., Kiritani, T., and Shepherd, G.M. (2010). Sublayer-specific microcircuits of corticospinal and corticostriatal neurons in motor cortex. Nat Neurosci 13, 739744.

Asanuma, H. (1981). Functional role of sensory inputs to the motor cortex. Progress in neurobiology 16, 241-262.

Atasoy, D., Aponte, Y., Su, H.H., and Sternson, S.M. (2008). A FLEX switch targets Channelrhodopsin-2 to multiple cell types for imaging and long-range circuit mapping. J Neurosci 28, 7025-7030.

Beierlein, M., Gibson, J.R., and Connors, B.W. (2003). Two dynamically distinct inhibitory networks in layer 4 of the neocortex. J Neurophysiol 90, 2987-3000.

Bock, D.D., Lee, W.C., Kerlin, A.M., Andermann, M.L., Hood, G., Wetzel, A.W., Yurgenson, S., Soucy, E.R., Kim, H.S., and Reid, R.C. (2011). Network anatomy and in vivo physiology of visual cortical neurons. Nature 471, 177-182.

Braitenberg, V.B., and Schuz, A. (1998). Cortex: statistics and geometry of neuronal connectivity (Berlin: Springer).

Bruno, R.M., and Simons, D.J. (2002). Feedforward mechanisms of excitatory and inhibitory cortical receptive fields. J Neurosci 22, 10966-10975.

Callaway, E.M., and Luo, L. (2015). Monosynaptic Circuit Tracing with Glycoprotein-Deleted Rabies Viruses. J Neurosci 35, 8979-8985.

Chakraibarti, S., and Alloway, K.D. (2006). Differential origin of projections from SI barrel cortex to the whisker representations in SII and MI. Journal of Comparative Neurology 498, 624-636. Chen, S.X., Kim, A.N., Peters, A.J., and Komiyama, T. (2015). Subtype-specific plasticity of inhibitory circuits in motor cortex during motor learning. Nat Neurosci 18, 1109-1115.

Cruikshank, S.J., Lewis, T.J., and Connors, B.W. (2007). Synaptic basis for intense thalamocortical activation of feedforward inhibitory cells in neocortex. Nat Neurosci $10,462-468$. 
592 Cruikshank, S.J., Urabe, H., Nurmikko, A.V., and Connors, B.W. (2010). Pathway-specific

593 feedforward circuits between thalamus and neocortex revealed by selective optical stimulation

594 of axons. Neuron 65, 230-245.

595 Deschenes, M., Veinante, P., and Zhang, Z.W. (1998). The organization of corticothalamic

596 projections: reciprocity versus parity. Brain Res Brain Res Rev 28, 286-308.

597 Dittrich, L., Heiss, J.E., Warrier, D.R., Perez, X.A., Quik, M., and Kilduff, T.S. (2012). Cortical

598 nNOS neurons co-express the NK1 receptor and are depolarized by Substance P in multiple

599 mammalian species. Front Neural Circuits 6, 31.

600 Dum, R.P., and Strick, P.L. (2002). Motor areas in the frontal lobe of the primate. Physiol Behav $60177,677-682$.

602 Eastwood, B.S., Hooks, B.M., Paletzki, R.F., O'Connor, N.J., Glaser, J.R., and Gerfen, C.R.

603 (2019). Whole mouse brain reconstruction and registration to a reference atlas with standard

604 histochemical processing of coronal sections. J Comp Neurol 527, 2170-2178.

605 Ferezou, I., Haiss, F., Gentet, L.J., Aronoff, R., Weber, B., and Petersen, C.C. (2007).

606 Spatiotemporal dynamics of cortical sensorimotor integration in behaving mice. Neuron 56, 907-

607923.

608 Fino, E., Packer, A.M., and Yuste, R. (2013). The logic of inhibitory connectivity in the 609 neocortex. Neuroscientist 19, 228-237.

610 Fino, E., and Yuste, R. (2011). Dense inhibitory connectivity in neocortex. Neuron 69, 11886111203.

612 Guo, K., Yamawaki, N., Barrett, J.M., Tapies, M., and Shepherd, G.M.G. (2020). Cortico-

613 Thalamo-Cortical Circuits of Mouse Forelimb S1 Are Organized Primarily as Recurrent Loops. J

614 Neurosci 40, 2849-2858.

615 Harris, J.A., Mihalas, S., Hirokawa, K.E., Whitesell, J.D., Choi, H., Bernard, A., Bohn, P.,

616 Caldejon, S., Casal, L., Cho, A., et al. (2019). Hierarchical organization of cortical and thalamic

617 connectivity. Nature 575, 195-202.

618 Harrison, T.C., and Murphy, T.H. (2014). Motor maps and the cortical control of movement. Curr

619 Opin Neurobiol 24, 88-94.

620 Hatsopoulos, N.G., and Suminski, A.J. (2011). Sensing with the motor cortex. Neuron 72, $477-$

621487.

622 Hill, D.N., Curtis, J.C., Moore, J.D., and Kleinfeld, D. (2011). Primary motor cortex reports

623 efferent control of vibrissa motion on multiple timescales. Neuron 72, 344-356. 
624 Hippenmeyer, S., Vrieseling, E., Sigrist, M., Portmann, T., Laengle, C., Ladle, D.R., and Arber,

625 S. (2005). A developmental switch in the response of DRG neurons to ETS transcription factor

626 signaling. PLoS Biol 3, e159.

627 Hoffer, Z.S., Hoover, J.E., and Alloway, K.D. (2003). Sensorimotor corticocortical projections

628 from rat barrel cortex have an anisotropic organization that facilitates integration of inputs from

629 whiskers in the same row. J Comp Neurol 466, 525-544.

630 Hooks, B.M. (2017). Sensorimotor Convergence in Circuitry of the Motor Cortex. Neuroscientist

$63123,251-263$.

632 Hooks, B.M., Lin, J.Y., Guo, C., and Svoboda, K. (2015). Dual-Channel Circuit Mapping Reveals

633 Sensorimotor Convergence in the Primary Motor Cortex. J Neurosci 35, 4418-4426.

634 Hooks, B.M., Mao, T., Gutnisky, D.A., Yamawaki, N., Svoboda, K., and Shepherd, G.M. (2013).

635 Organization of cortical and thalamic input to pyramidal neurons in mouse motor cortex. $\mathrm{J}$

636 Neurosci 33, 748-760.

637 Hooks, B.M., Papale, A.E., Paletzki, R.F., Feroze, M.W., Eastwood, B.S., Couey, J.J.,

638 Winnubst, J., Chandrashekar, J., and Gerfen, C.R. (2018). Topographic precision in sensory

639 and motor corticostriatal projections varies across cell type and cortical area. Nat Commun 9 , 6403549.

641 Huang, H., Kuzirian, M.S., Cai, X., Snyder, L.M., Cohen, J., Kaplan, D.H., and Ross, S.E.

642 (2016). Generation of a NK1R-CreER knockin mouse strain to study cells involved in Neurokinin

6431 Receptor signaling. Genesis 54, 593-601.

644 Huber, D., Gutnisky, D.A., Peron, S., O'Connor, D.H., Wiegert, J.S., Tian, L., Oertner, T.G., 645 Looger, L.L., and Svoboda, K. (2012). Multiple dynamic representations in the motor cortex

646 during sensorimotor learning. Nature 484, 473-478.

647 Isaacson, J.S., and Scanziani, M. (2011). How inhibition shapes cortical activity. Neuron 72,

648 231-243.

649 Jackman, S.L., Beneduce, B.M., Drew, I.R., and Regehr, W.G. (2014). Achieving high-

650 frequency optical control of synaptic transmission. J Neurosci 34, 7704-7714.

651 Kaneko, T., Caria, M.A., and Asanuma, H. (1994a). Information processing within the motor

652 cortex. I. Responses of morphologically identified motor cortical cells to stimulation of the

653 somatosensory cortex. J Comp Neurol 345, 161-171.

654 Kaneko, T., Caria, M.A., and Asanuma, H. (1994b). Information processing within the motor

655 cortex. II. Intracortical connections between neurons receiving somatosensory cortical input and 656 motor output neurons of the cortex. J Comp Neurol 345, 172-184. 
Katzel, D., Zemelman, B.V., Buetfering, C., Wolfel, M., and Miesenbock, G. (2011). The columnar and laminar organization of inhibitory connections to neocortical excitatory cells. Nat Neurosci 14, 100-107.

Kinnischtzke, A.K., Simons, D.J., and Fanselow, E.E. (2014). Motor cortex broadly engages excitatory and inhibitory neurons in somatosensory barrel cortex. Cereb Cortex 24, 2237-2248. Kiritani, T., Wickersham, I.R., Seung, H.S., and Shepherd, G.M. (2012). Hierarchical connectivity and connection-specific dynamics in the corticospinal-corticostriatal microcircuit in mouse motor cortex. J Neurosci 32, 4992-5001.

Klapoetke, N.C., Murata, Y., Kim, S.S., Pulver, S.R., Birdsey-Benson, A., Cho, Y.K., Morimoto, T.K., Chuong, A.S., Carpenter, E.J., Tian, Z., et al. (2014). Independent optical excitation of distinct neural populations. Nat Methods 11, 338-346.

Kuan, L., Li, Y., Lau, C., Feng, D., Bernard, A., Sunkin, S.M., Zeng, H., Dang, C., Hawrylycz, M., and $\mathrm{Ng}$, L. (2015). Neuroinformatics of the Allen Mouse Brain Connectivity Atlas. Methods 73, 417.

Kubota, Y., Shigematsu, N., Karube, F., Sekigawa, A., Kato, S., Yamaguchi, N., Hirai, Y., Morishima, M., and Kawaguchi, Y. (2011). Selective coexpression of multiple chemical markers defines discrete populations of neocortical GABAergic neurons. Cereb Cortex 21, 1803-1817. Kuramoto, E., Furuta, T., Nakamura, K.C., Unzai, T., Hioki, H., and Kaneko, T. (2009). Two types of thalamocortical projections from the motor thalamic nuclei of the rat: a single neurontracing study using viral vectors. Cereb Cortex 19, 2065-2077.

Kuramoto, E., Ohno, S., Furuta, T., Unzai, T., Tanaka, Y.R., Hioki, H., and Kaneko, T. (2015). Ventral medial nucleus neurons send thalamocortical afferents more widely and more preferentially to layer 1 than neurons of the ventral anterior-ventral lateral nuclear complex in the rat. Cereb Cortex 25, 221-235.

Lee, S., Hjerling-Leffler, J., Zagha, E., Fishell, G., and Rudy, B. (2010). The largest group of superficial neocortical GABAergic interneurons expresses ionotropic serotonin receptors. J Neurosci 30, 16796-16808.

Ma, Y., Hu, H., Berrebi, A.S., Mathers, P.H., and Agmon, A. (2006). Distinct subtypes of somatostatin-containing neocortical interneurons revealed in transgenic mice. J Neurosci 26, 5069-5082.

Madisen, L., Zwingman, T.A., Sunkin, S.M., Oh, S.W., Zariwala, H.A., Gu, H., Ng, L.L., Palmiter, R.D., Hawrylycz, M.J., Jones, A.R., et al. (2010). A robust and high-throughput Cre reporting and characterization system for the whole mouse brain. Nat Neurosci 13, 133-140. 
Mao, T., Kusefoglu, D., Hooks, B.M., Huber, D., Petreanu, L., and Svoboda, K. (2011). Longrange neuronal circuits underlying the interaction between sensory and motor cortex. Neuron 72, 111-123.

Masamizu, Y., Tanaka, Y.R., Tanaka, Y.H., Hira, R., Ohkubo, F., Kitamura, K., Isomura, Y., Okada, T., and Matsuzaki, M. (2014). Two distinct layer-specific dynamics of cortical ensembles during learning of a motor task. Nat Neurosci 17, 987-994.

Muñoz-Castaneda, R.Z., B.; Matho, K. S.; Wang, Q.; Chen, X.; et al. (2020). Cellular Anatomy of the Mouse Primary Motor Cortex. bioRxiv.

Murray, P.D., and Keller, A. (2011). Somatosensory response properties of excitatory and inhibitory neurons in rat motor cortex. J Neurophysiol 106, 1355-1362.

Naskar, S., Qi, J., Pereira, F., Gerfen, C.R., and Lee, S. (2021). Cell-type-specific recruitment of GABAergic interneurons in the primary somatosensory cortex by long-range inputs. Cell Rep 34, 108774.

Oh, S.W., Harris, J.A., Ng, L., Winslow, B., Cain, N., Mihalas, S., Wang, Q., Lau, C., Kuan, L., Henry, A.M., et al. (2014). A mesoscale connectome of the mouse brain. Nature 508, 207-214. Ohno, S., Kuramoto, E., Furuta, T., Hioki, H., Tanaka, Y.R., Fujiyama, F., Sonomura, T., Uemura, M., Sugiyama, K., and Kaneko, T. (2012). A morphological analysis of thalamocortical axon fibers of rat posterior thalamic nuclei: a single neuron tracing study with viral vectors. Cereb Cortex 22, 2840-2857.

Packer, A.M., and Yuste, R. (2011). Dense, unspecific connectivity of neocortical parvalbuminpositive interneurons: a canonical microcircuit for inhibition? J Neurosci 31, 13260-13271. Petilla Interneuron Nomenclature, G., Ascoli, G.A., Alonso-Nanclares, L., Anderson, S.A., Barrionuevo, G., Benavides-Piccione, R., Burkhalter, A., Buzsaki, G., Cauli, B., Defelipe, J., et al. (2008). Petilla terminology: nomenclature of features of GABAergic interneurons of the cerebral cortex. Nat Rev Neurosci 9, 557-568.

Petreanu, L., Huber, D., Sobczyk, A., and Svoboda, K. (2007). Channelrhodopsin-2-assisted circuit mapping of long-range callosal projections. Nat Neurosci 10, 663-668.

Petreanu, L., Mao, T., Sternson, S.M., and Svoboda, K. (2009). The subcellular organization of neocortical excitatory connections. Nature 457, 1142-1145.

Pfeffer, C.K., Xue, M., He, M., Huang, Z.J., and Scanziani, M. (2013). Inhibition of inhibition in visual cortex: the logic of connections between molecularly distinct interneurons. Nat Neurosci 16, 1068-1076.

Porter, J.T., Johnson, C.K., and Agmon, A. (2001). Diverse types of interneurons generate thalamus-evoked feedforward inhibition in the mouse barrel cortex. J Neurosci 21, 2699-2710. 
Reardon, T.R., Murray, A.J., Turi, G.F., Wirblich, C., Croce, K.R., Schnell, M.J., Jessell, T.M., and Losonczy, A. (2016). Rabies Virus CVS-N2c(DeltaG) Strain Enhances Retrograde Synaptic Transfer and Neuronal Viability. Neuron 89, 711-724.

Reep, R.L., Goodwin, G.S., and Corwin, J.V. (1990). Topographic organization in the corticocortical connections of medial agranular cortex in rats. J Comp Neurol 294, 262-280. Rouiller, E.M., Moret, V., and Liang, F. (1993). Comparison of the connectional properties of the two forelimb areas of the rat sensorimotor cortex: support for the presence of a premotor or supplementary motor cortical area. Somatosens Mot Res 10, 269-289.

Shepherd, G.M. (2013). Corticostriatal connectivity and its role in disease. Nat Rev Neurosci 14, 278-291.

Shepherd, G.M., Pologruto, T.A., and Svoboda, K. (2003). Circuit analysis of experiencedependent plasticity in the developing rat barrel cortex. Neuron 38, 277-289.

Sherman, S.M. (2001). Tonic and burst firing: dual modes of thalamocortical relay. Trends Neurosci 24, 122-126.

Smith, J.B., and Alloway, K.D. (2013). Rat whisker motor cortex is subdivided into sensory-input and motor-output areas. Front Neural Circuits 7, 4.

Steriade, M., McCormick, D.A., and Sejnowski, T.J. (1993). Thalamocortical oscillations in the sleeping and aroused brain. Science 262, 679-685.

Strick, P.L., and Sterling, P. (1974). Synaptic termination of afferents from the ventrolateral nucleus of the thalamus in the cat motor cortex. A light and electron microscopy study. J Comp Neurol 153, 77-106.

Suter, B.A., O'Connor, T., Iyer, V., Petreanu, L.T., Hooks, B.T., Kiritani, T., Svoboda, K., and G.M.G, S. (2010). Ephus: multipurpose data acquisition software for neuroscience experiments. Frontiers Neurosci 4.

Suter, B.A., and Shepherd, G.M. (2015). Reciprocal interareal connections to corticospinal neurons in mouse M1 and S2. J Neurosci 35, 2959-2974.

Tan, Z., Hu, H., Huang, Z.J., and Agmon, A. (2008). Robust but delayed thalamocortical activation of dendritic-targeting inhibitory interneurons. Proc Natl Acad Sci U S A 105, 21872192.

Tanaka, Y.H., Tanaka, Y.R., Kondo, M., Terada, S.I., Kawaguchi, Y., and Matsuzaki, M. (2018). Thalamocortical Axonal Activity in Motor Cortex Exhibits Layer-Specific Dynamics during Motor Learning. Neuron 100, 244-258 e212. 
Taniguchi, H., He, M., Wu, P., Kim, S., Paik, R., Sugino, K., Kvitsiani, D., Fu, Y., Lu, J., Lin, Y., et al. (2011). A resource of Cre driver lines for genetic targeting of GABAergic neurons in cerebral cortex. Neuron 71, 995-1013.

Tasic, B., Yao, Z., Graybuck, L.T., Smith, K.A., Nguyen, T.N., Bertagnolli, D., Goldy, J., Garren, E., Economo, M.N., Viswanathan, S., et al. (2018). Shared and distinct transcriptomic cell types across neocortical areas. Nature 563, 72-78.

Tremblay, R., Lee, S., and Rudy, B. (2016). GABAergic Interneurons in the Neocortex: From Cellular Properties to Circuits. Neuron 91, 260-292.

Tuncdemir, S.N., Wamsley, B., Stam, F.J., Osakada, F., Goulding, M., Callaway, E.M., Rudy, B., and Fishell, G. (2016). Early Somatostatin Interneuron Connectivity Mediates the Maturation of Deep Layer Cortical Circuits. Neuron 89, 521-535.

Weiler, N., Wood, L., Yu, J., Solla, S.A., and Shepherd, G.M. (2008). Top-down laminar organization of the excitatory network in motor cortex. Nat Neurosci 11, 360-366.

Wickersham, I.R., Lyon, D.C., Barnard, R.J., Mori, T., Finke, S., Conzelmann, K.K., Young, J.A., and Callaway, E.M. (2007). Monosynaptic restriction of transsynaptic tracing from single, genetically targeted neurons. Neuron 53, 639-647.

Xue, M., Atallah, B.V., and Scanziani, M. (2014). Equalizing excitation-inhibition ratios across visual cortical neurons. Nature 511, 596-600.

Yamawaki, N., Radulovic, J., and Shepherd, G.M. (2016). A Corticocortical Circuit Directly Links Retrosplenial Cortex to M2 in the Mouse. J Neurosci 36, 9365-9374.

Zeisel, A., Munoz-Manchado, A.B., Codeluppi, S., Lonnerberg, P., La Manno, G., Jureus, A., Marques, S., Munguba, H., He, L., Betsholtz, C., et al. (2015). Brain structure. Cell types in the mouse cortex and hippocampus revealed by single-cell RNA-seq. Science 347, 1138-1142.

Zingg, B., Hintiryan, H., Gou, L., Song, M.Y., Bay, M., Bienkowski, M.S., Foster, N.N., Yamashita, S., Bowman, I., Toga, A.W., et al. (2014). Neural networks of the mouse neocortex. Cell 156, 1096-1111. 
Figure 1 | Mapping long-range excitatory inputs to mouse motor cortex interneurons

(a) How do interneuron populations carry feedforward inhibition in M1? Whole-cell recordings at -70 mV (black) and 0mV (red) show optically induced feedforward inhibition from S1 inputs to M1 (left). EPSC and IPSC amplitude are roughly proportional (right) across all cortical layers. (b) AAV vectors expressing optogenetic activators (ChR2 or Chronos) were stereotaxically injected into cortex (S1, light green) or thalamus (PO, dark green). (c) mVenus+ axons from S1 arborize across layers of M1 in a columnar projection (left, 4x image). Fluorescence is highest in L5A (right, brightness quantified across cortical layers). (d) mVenus+ axons from PO arborize across layers of M1 in two main layers (left). Fluorescence is highest in L1 and L5A (right). (e) PV-Cre mice were crossed to a tdTomato reporter (Ai14), labeling interneurons across M1 in L2-6. Postsynaptic interneurons were recorded across all layers for comparison. Inset (60x, fluorescence image, top, and corresponding brightfield image, bottom) shows patch pipette on tdTomato+ neuron in PV-Cre x Ai14 mouse. (f) SOM-Cre mice were crossed to a tdTomato reporter (Ai14), labeling interneurons across M1 in L2-6. L1 signal represents tdTomato+ axons from SOM+ neurons. Postsynaptic interneurons were recorded across all layers for comparison. stimulation excites axons at example points $1 \& 2$, but TTX addition extinguishes responses. Addition of 4-AP permits local release at points where opsin+ axons contact interneuron dendrites (Point 1) but not distant points along the axon (Point 2). (i) Similar concentrations of 4AP are required to restore synaptic responses regardless of postsynaptic neuron type (Pyr =

813 pyramidal neuron). Individual cells plotted at left; average and SE plotted at right. Grey box

814 represents [4-AP] used for mapping. (j) Typical input mapping experiments sampled a 12x26

815 point grid with points spaced at $50 \mathrm{~mm}$ and aligned to the pia. Brightfield image (4x) shows

816 recording pipette in M1. (k) Heatmap shows stronger responses (red) near the soma of the

817 recorded neuron. (I) Example traces for a $L 2 / 3$ PV+ neuron show location of input. (m) Inset

818 shows two maps to compare input between L2/3 and L5A PV+ interneurons. The summed

819 synaptic input across the map is compared for each cell pair recorded in the same slice. Arrow

820 indicates a point from one L2/3-L5A pair. Dashed line represents $y=x$ (similar input to both

821 layers); solid line represents the geometric mean of the L2/3 and L5A input strength, presented

822 as a line of equivalent slope. 
824

825

826

827

828

829

830

831

832

833

834

835

836

837

838

839

840

841

842

843

844

845

846

847

848

849

850

851

852

853

854

855

856

857

\section{Figure 2 | S1 input to M1 targets upper layer PV+ neurons}

(a) Cartoon showing PV+ neurons (red) in GFP+ axons from S1. (b) S1 input to M1 PV+ neurons across layers ( $\mathrm{N}=58$ total). Each cell is represented as a single column (vector), with the rows of the input mapped summed and aligned to the pia. Diagonal white line represents the soma location for that cell. Vertical white lines represent layer divisions. Synaptic strength (summed, in $\mathrm{nA}$ ) for each location in depth are represented by the heatmap (scale at right). Laminar depth is normalized to $0=$ pia, $1=$ white matter. (c) Strength of synaptic input. The bar represents the geometric means of the amplitude ratio, normalized to the layer receiving the strongest input (L2/3). The overlaid graph shows the mean ratio and SD (based on 10000 replicate bootstrap). Adjacent to the summary, three graphs for comparison of input strength across neurons in different cortical layers. Each point represents input to a pair of neurons in the same slice (circle for each neuron). Dashed line represents unity. N, Number of pairs; $p$ value, Wilcoxon signed rank test. (d) Maps of synaptic input location in the dendritic arbor for PV+ neurons in each layer. Top row, normalized soma-centered map (maps registered to soma center across cells). Bottom row, normalized pia-aligned maps. Normalized maps are noisy when input is weak. (e,f) Input location summarized for all four layers. Normalized mean input maps were averaged into a vector (e) and aligned to the soma (red circle). These were graphed with mean and SD (f), showing input relative to the soma in $50 \mathrm{~mm}$ bins. Dashed line indicates soma depth. ${ }^{*}=p<0.05,{ }^{* *}=p<0.01$, and ${ }^{* * *}=p<0.001$.

\section{Figure 3 | S1 input to M1 targets middle layer SOM+ neurons}
(a) Cartoon showing SOM+ neurons (red) in GFP+ axons from S1. (b) S1 input to SOM+ neurons across layers ( $\mathrm{N}=53$ total). Each cell is represented as a single column (vector), with the rows of the input mapped summed and aligned to the pia. Diagonal white line represents the soma location for that cell. Vertical white lines represent layer divisions. Synaptic strength (summed, in $\mathrm{nA}$ ) for each location in depth are represented by the heatmap (scale at right). Laminar depth is normalized to $0=$ pia, $1=$ white matter. (c) Strength of synaptic input. The bar represents the geometric means of the amplitude ratio, normalized to the layer receiving the strongest input (L5A). The overlaid graph shows the mean ratio and SD (based on 10000 replicate bootstrap). Adjacent to the summary, three graphs for comparison of input strength across neurons in different cortical layers. Each point represents input to a pair of neurons in the same slice (circle for each neuron). Dashed line represents unity. N, Number of pairs; $p$ value, Wilcoxon signed rank test. (d) Maps of synaptic input location in the dendritic arbor for SOM+ neurons in each layer. Top row, normalized soma-centered map (maps registered to soma 
center across cells). Bottom row, normalized pia-aligned maps. Normalized maps are noisy when input is weak. (e,f) Input location summarized for all four layers. Normalized mean input maps were averaged into a vector (e) and aligned to the soma (red circle). These were graphed with mean and SD (f), showing input relative to the soma in $50 \mathrm{~mm}$ bins. Dashed line indicates soma depth.

\section{Figure 4 | PO input to M1 PV+ neurons is targets upper and middle layers} (a) Cartoon showing PV+ neurons (red) in GFP+ axons from PO. (b) PO input to PV+ neurons across layers ( $\mathrm{N}=61$ total). Each cell is represented as a single column (vector), with the rows of the input mapped summed and aligned to the pia. Diagonal white line represents the soma location for that cell. Vertical white lines represent layer divisions. Synaptic strength (summed, in $\mathrm{nA}$ ) for each location in depth are represented by the heatmap (scale at right). Laminar depth is normalized to $0=$ pia, $1=$ white matter. (c) Strength of synaptic input. The bar represents the geometric means of the amplitude ratio, normalized to the layer receiving the strongest input (L5A). The overlaid graph shows the mean ratio and SD (based on 10000 replicate bootstrap). Adjacent to the summary, three graphs for comparison of input strength across neurons in different cortical layers. Each point represents input to a pair of neurons in the same slice (circle for each neuron). Dashed line represents unity. N, Number of pairs; $p$ value, Wilcoxon signed rank test. (d) Maps of synaptic input location in the dendritic arbor for $\mathrm{PV}+$ neurons in each layer. Top row, normalized soma-centered map (maps registered to soma center across cells). Bottom row, normalized pia-aligned maps. Normalized maps are noisy when input is weak. (e,f) Input location summarized for all four layers. Normalized mean input maps were averaged into a vector (e) and aligned to the soma (red circle). These were graphed with mean and SD (f), showing input relative to the soma in $50 \mathrm{~mm}$ bins. Dashed line indicates soma depth.

\section{Figure 5 | PO input to M1 SOM+ neurons is variable}

(a) Cartoon showing SOM+ neurons (red) in GFP+ axons from PO. (b) PO input to SOM+ neurons across layers ( $\mathrm{N}=48$ total). Each cell is represented as a single column (vector), with the rows of the input mapped summed and aligned to the pia. Diagonal white line represents the soma location for that cell. Vertical white lines represent layer divisions. Synaptic strength (summed, in $n A$ ) for each location in depth are represented by the heatmap (scale at right). Laminar depth is normalized to $0=$ pia, $1=$ white matter. (c) Strength of synaptic input. The bar represents the geometric means of the amplitude ratio, normalized to the layer receiving the strongest input (L5B). The overlaid graph shows the mean ratio and SD (based on 10000 
replicate bootstrap). Adjacent to the summary, three graphs for comparison of input strength across neurons in different cortical layers. Each point represents input to a pair of neurons in the same slice (circle for each neuron). Dashed line represents unity. N, Number of pairs; $p$ value, Wilcoxon signed rank test. Plots show substantial heterogeneity. (d) Maps of synaptic input location in the dendritic arbor for SOM+ neurons in each layer. Top row, normalized somacentered map (maps registered to soma center across cells). Bottom row, normalized piaaligned maps. Normalized maps are noisy when input is weak. (e,f) Input location summarized for all four layers. Normalized mean input maps were averaged into a vector (e) and aligned to the soma (red circle). These were graphed with mean and SD (f), showing input relative to the soma in $50 \mathrm{~mm}$ bins. Dashed line indicates soma depth.

\section{Figure 6 | Complementary patterns of input to specific interneuron types}

(a-d) Summary graphs comparing the distribution of excitatory input to $\mathrm{PV}+$ and $\mathrm{SOM}+$ neurons.

These plots compare normalized input from (a) S1 only or (b) PO only to PV+ and SOM+ neurons. (c-d) compare input to PV+ only (c) or SOM+ only interneurons. (e) The pattern of normalized input strength to pyramidal neurons (Pyr) from S1 and PO, after Mao et al (2011) and Hooks et al. (2013). (f) Cartoon summarizing input maps. (g) Absolute input strength for S1 and PO inputs, plotted as input vectors on a log scale. Top row, PV+ neurons; middle row, $\mathrm{SOM}+$ neurons, bottom row, Tacr1+ neurons (a subset of SOM+ neurons). Left column, S1 inputs. Right column, PO inputs. Data presented as in Figs 2-5. For Tacr1+ neurons, N=3/2/4/4 for $S 1$ input to $L 2 / 3, L 5 A, L 5 B$, and L6; $N=3 / 2 / 9 / 2$ for $P O$ input. (h) Absolute input strength averaged by layer and plotted on a log scale for comparison between S1 and PO inputs to different interneuron types (left column) or input from S1 or PO (right column).

\section{Figure 7 | Short-term plasticity at long range inputs to interneurons of mouse motor cortex}

(a) Short term plasticity of S1 input to PV+ neurons. Left, example traces showing PV+ neuron response to $\mathrm{S} 1$ stimulation at $10 \mathrm{~Hz}$ (red), $20 \mathrm{~Hz}$ (green), and $40 \mathrm{~Hz}$ (blue) stimulation of Chronos-GFP+ S1 axons. Scale, $50 \mathrm{pA}$ and $100 \mathrm{~ms}$. Middle, average normalized traces for the same stimulus frequencies. Scale bar=1.0. Right, summary graphs showing normalized responses for $10 \mathrm{~Hz}, 20 \mathrm{~Hz}$, and $40 \mathrm{~Hz}$. Graphs are plotted based on time of the pulses (right) or the stimulus number (far right). (b) Short term plasticity of PO input to PV+ neurons, plotted as in (a). (c) Short term plasticity of S1 input to SOM+ neurons, plotted as in (a). (d) Short term plasticity of PO input to SOM+ neurons, plotted as in (a). 
Figure 8 | Monosynaptic Retrograde Tracing of Inputs to Motor Cortex PV+ and SOM+ Neurons pseudotyped N2c RABV-tdTomato. (a) Low magnification image. Blue, Neurotrace; Green, TVA+ neurons (putative starter cells); Red, tdTomato+ neurons (putative presynaptic neurons). (b) Merged image of green (c) and red (d) channels, showing yellow starter cells (arrowheads) and singly labeled cells (blue arrows in c and yellow arrows in d). (e) Coronal section showing the Allen CCF 3.0 and locations of S1 and motor and somatosensory thalamic nuclei (PO, posterior nuc. of thalamus; S1, primary somatosensory cortex; VL, ventrolateral nuc.; VM, ventromedial nuc.; VPL, ventroposterolateral nuc.; VPM, ventroposteromedial nuc.). The positions of retrogradely labeled presynaptic neurons are marked with a red dot (top image) in an example SOM-Cre mouse injected in M1. Presynaptic neurons within $\pm 0.1 \mathrm{~mm}$ of the plane are shown. The bottom image (more posterior plane) is labeled to indicate cortical and thalamic areas. ( $\mathrm{f}-\mathrm{g}$ ) Example section showing retrograde label in $\mathrm{S} 1$ and thalamus from $\mathrm{M} 1$ starter cells in a PV-Cre mouse. Note S1 label and intense thalamic label. (h-i) Example section showing retrograde label in S1 and thalamus from M1 starter cells in a SOM-Cre mouse. Note S1 label and intense thalamic label. (j) Quantification of presynaptic neuron location. Whole brain image stacks were aligned to the Allen CCF V3.0. Presynaptic neuronal somata (red cells) were detected and coordinates in CCF assigned to brain regions. Fraction of the total presynaptic neurons population plotted for all motor cortex injections in PV-Cre ( $N=5$, red) and SOM-Cre $(\mathrm{N}=2$, blue) mice. Dotted lines represent individual cases; solid line represents mean. $(\mathrm{k})$ Quantification of thalamic inputs for all motor and frontal injections in PV-Cre $(\mathrm{N}=5$, red) and SOM-Cre ( $\mathrm{N}=2$, blue) mice. Data plotted as in (j), subdivided into distinct thalamic nuclei.

\section{Supplemental Figure 1 | Short-term plasticity comparisons of PV+ interneurons and}

952 pyramidal neurons

953 (a) Short term plasticity of S1 input to pyramidal neurons. Left, normalized response to S1 954 stimulation at $10 \mathrm{~Hz}$ (red), $20 \mathrm{~Hz}$ (green), and $40 \mathrm{~Hz}$ (blue) stimulation of Chronos-GFP+.

955 Summary graphs show responses plotted based on time of the pulses (left) or the stimulus 956 number (right). (b) Short term plasticity of PO input to pyramidal neurons, plotted as in (a). (c,d) 957 Short term plasticity of S1 input (c) and PO input (d) to PV+ interneurons plotted for comparison.

958 (e,f) Comparison plot of the above data for $10 \mathrm{~Hz}$ and $40 \mathrm{~Hz}$ stimulation on the same axes. 
960 Supplemental Figure 2 | Monosynaptic Retrograde Tracing of Inputs to Cortical PV+ and 961 SOM+ Neurons

962 (a-b) Quantification of presynaptic neuron location for injections in SOM-Cre (N=2, M2; N=2,

$963 \mathrm{fM} 1 ; \mathrm{N}=1$, vS1; cool colors) mice. Whole brain image stacks were aligned to the Allen CCF

964 V3.0. Presynaptic neuronal somata were detected and coordinates in CCF assigned to brain

965 regions. Mean fraction of the total presynaptic neurons population plotted as in Fig. 8.

966 Quantification of inptus to all brain regions (a) and to thalamic nuclei (b). (c-d) Data from

967 injections in PV-Cre ( $N=2, M 2 ; N=5, f M 1 ; N=1$, vS1; hot colors) plotted as in (a-b). (e-j) Data of

968 (a-d) reploteed to compare injections tracing input to SOM+ and $\mathrm{PV}+$ neurons in the same

969 cortical locations: M2 (e-f), fM1 (g-h), and vS1 (i-j).

970 
a

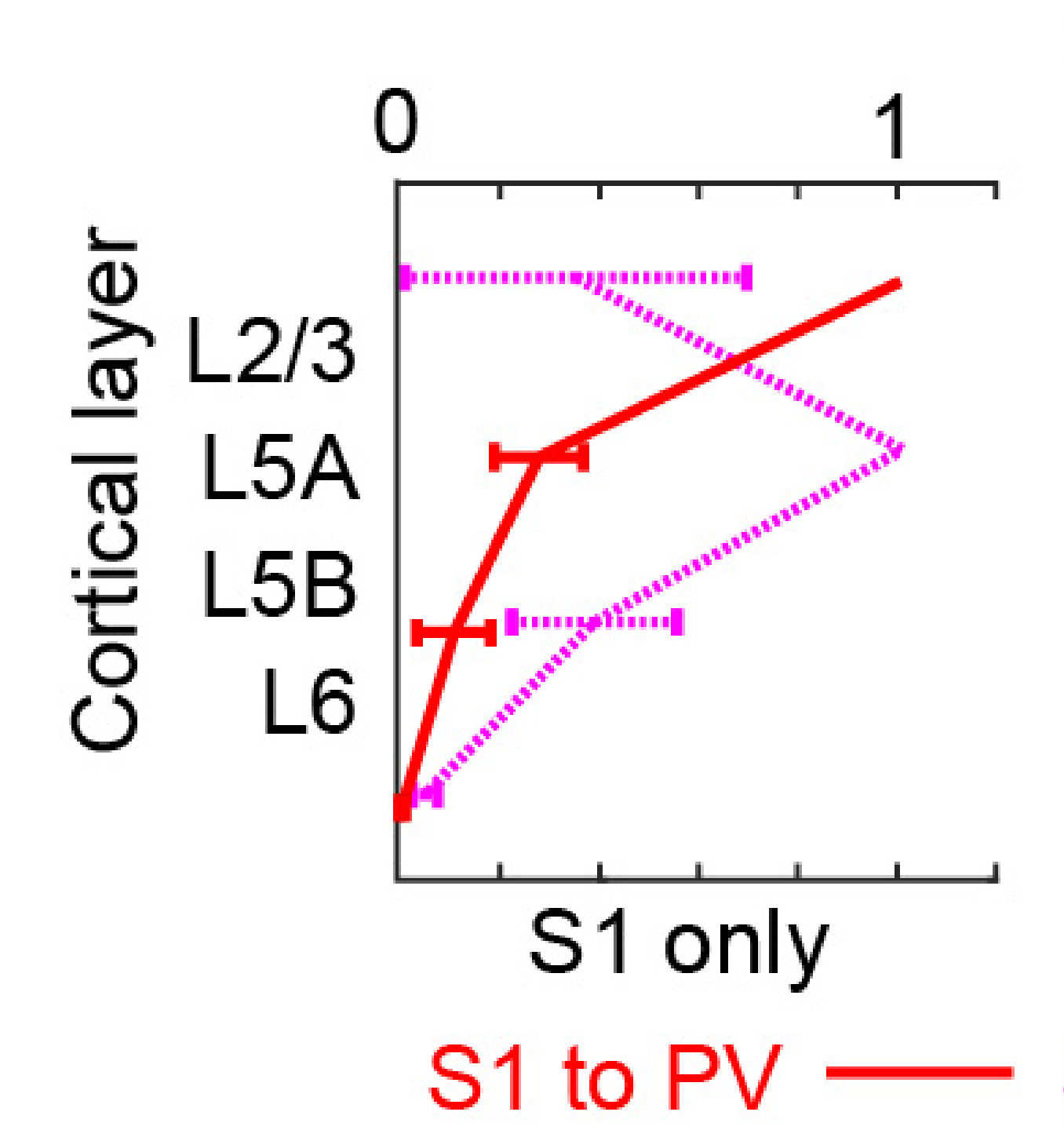

Normalized Input to Interneurons

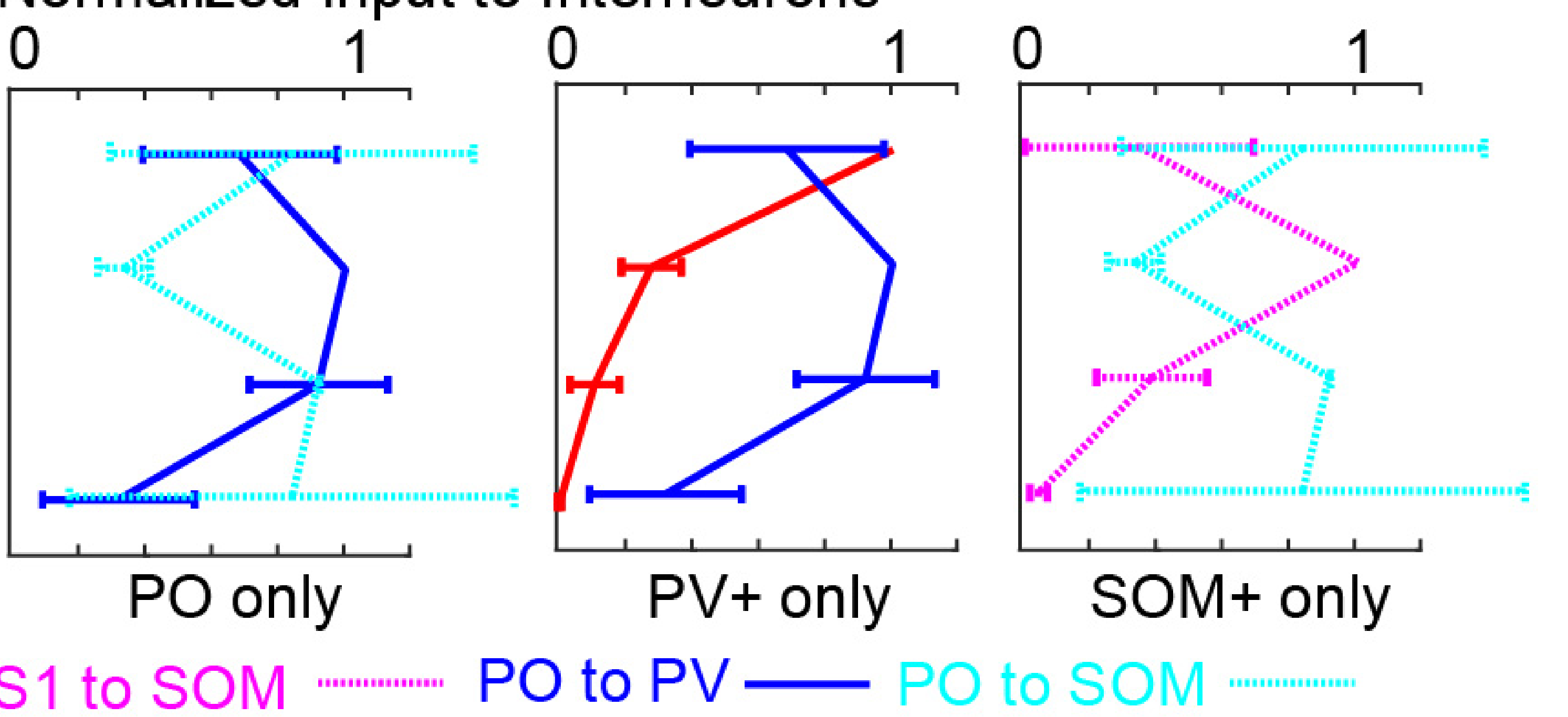

e Normalized Input $f$ to Pyramids

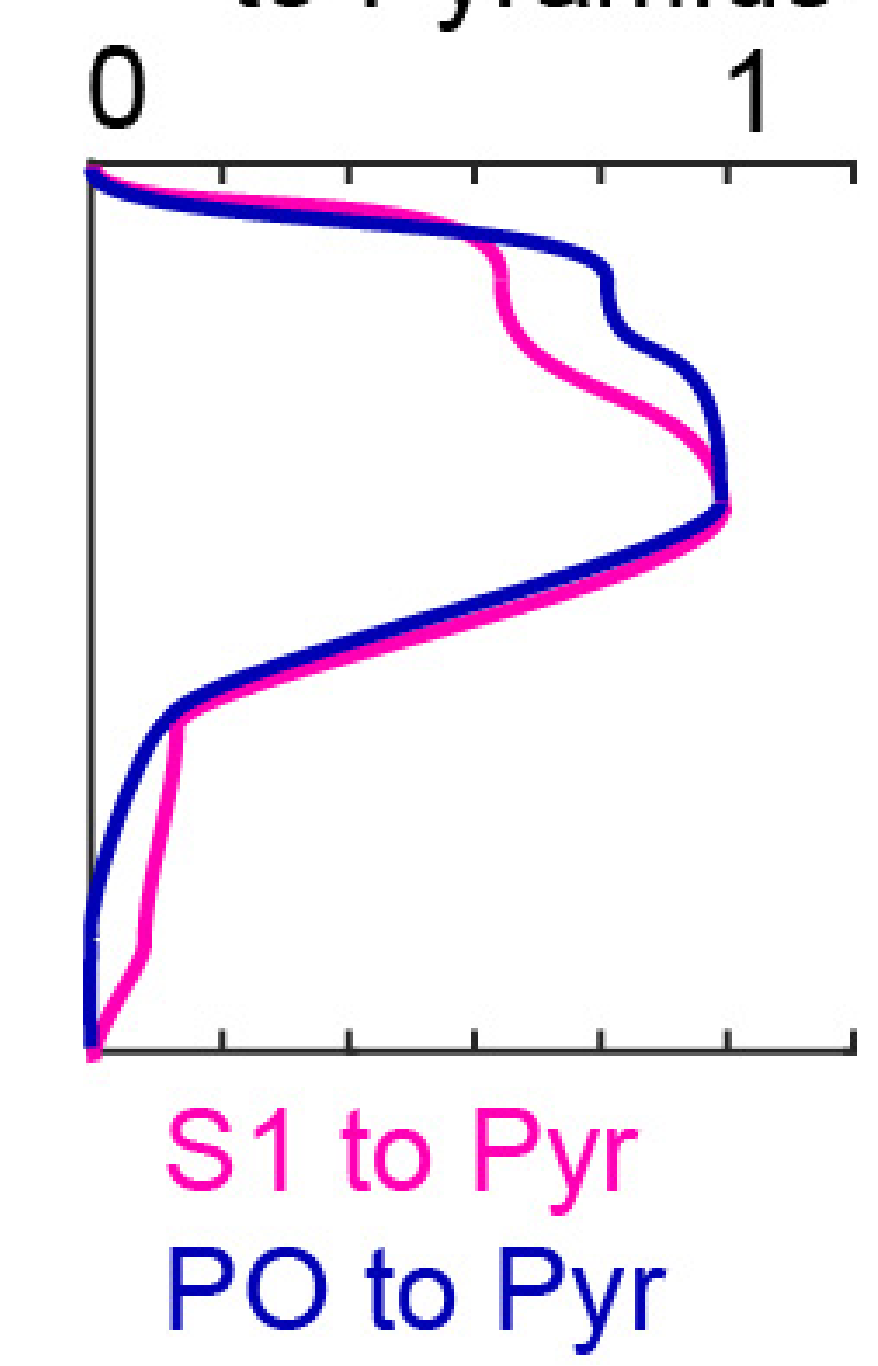

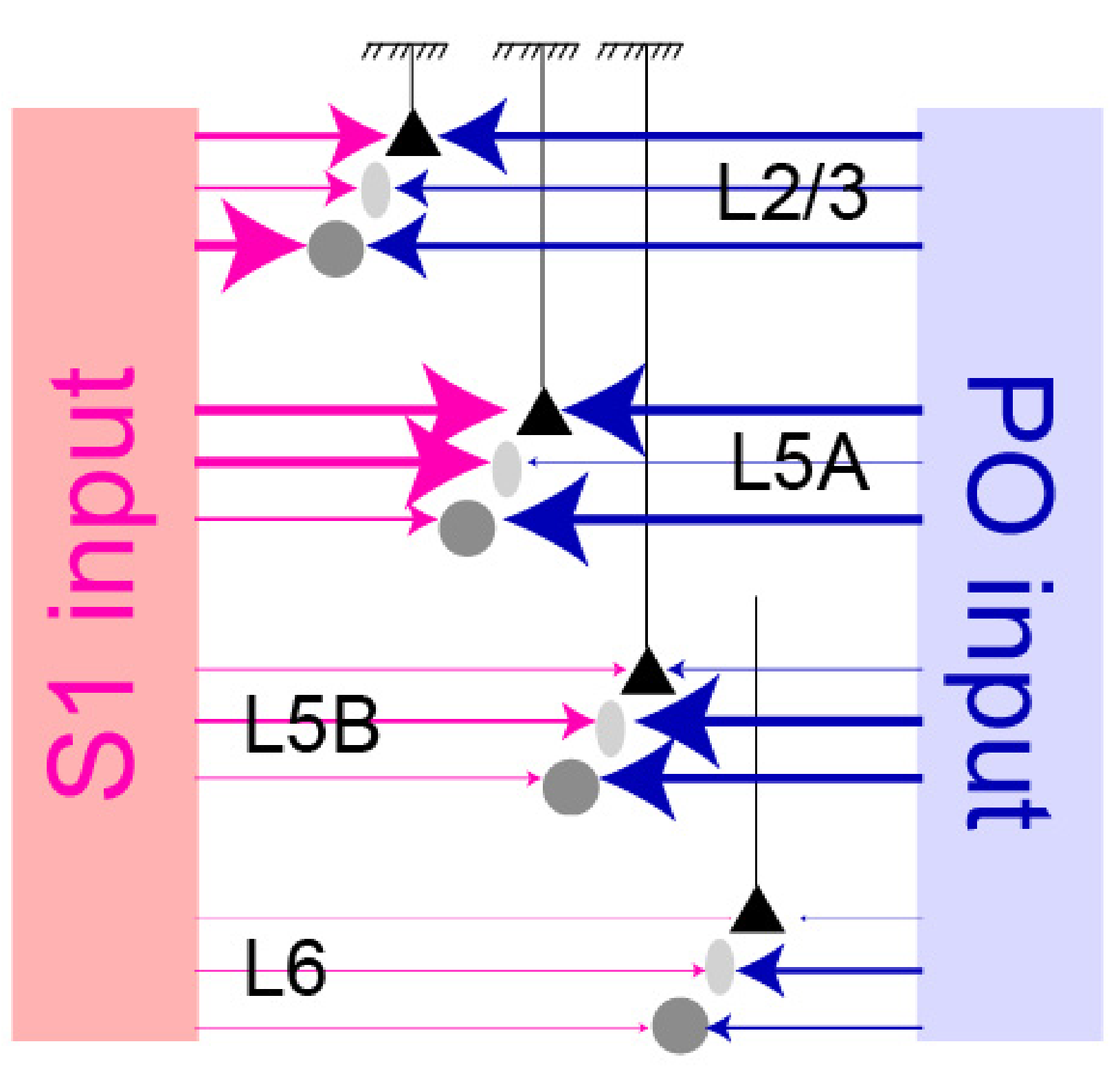

$\Delta$ Pyramid g

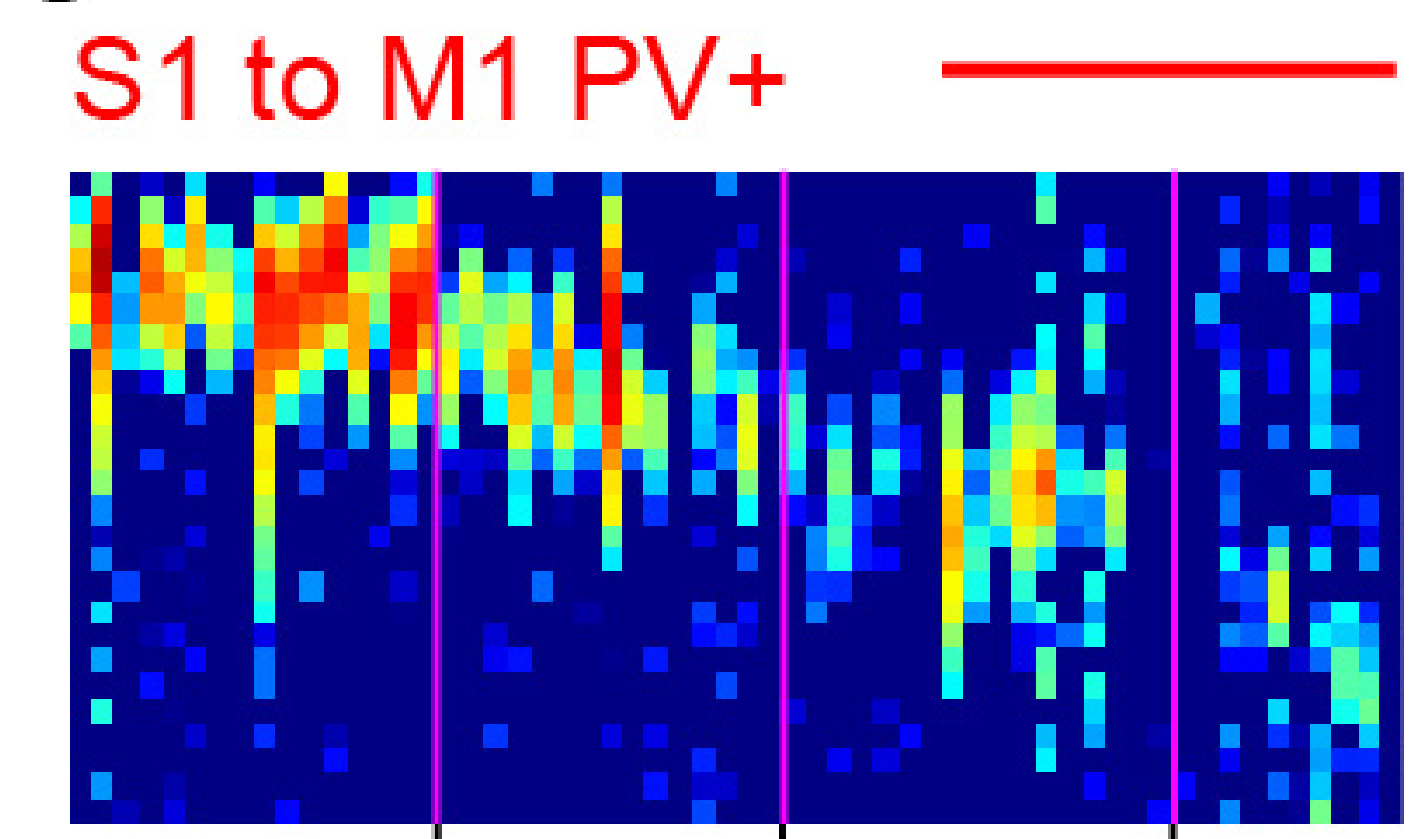

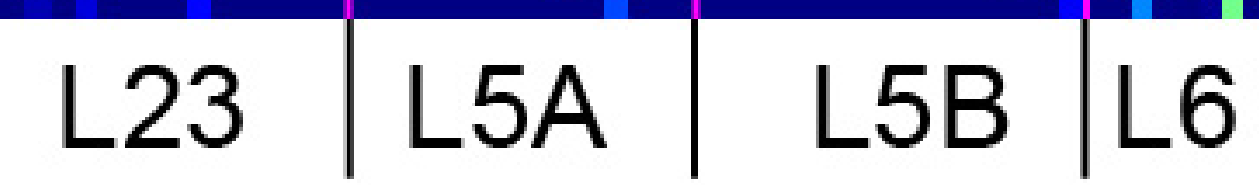

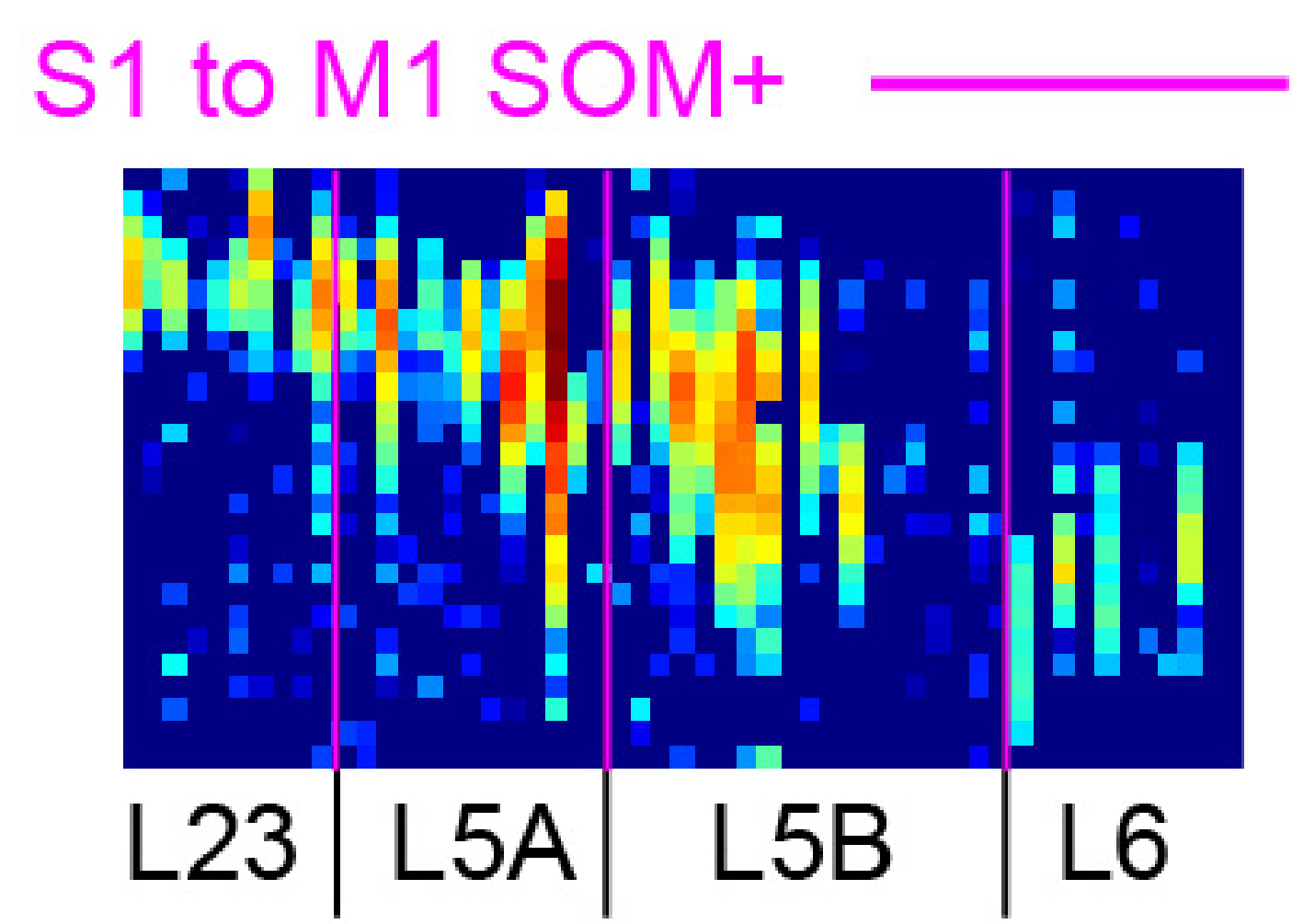

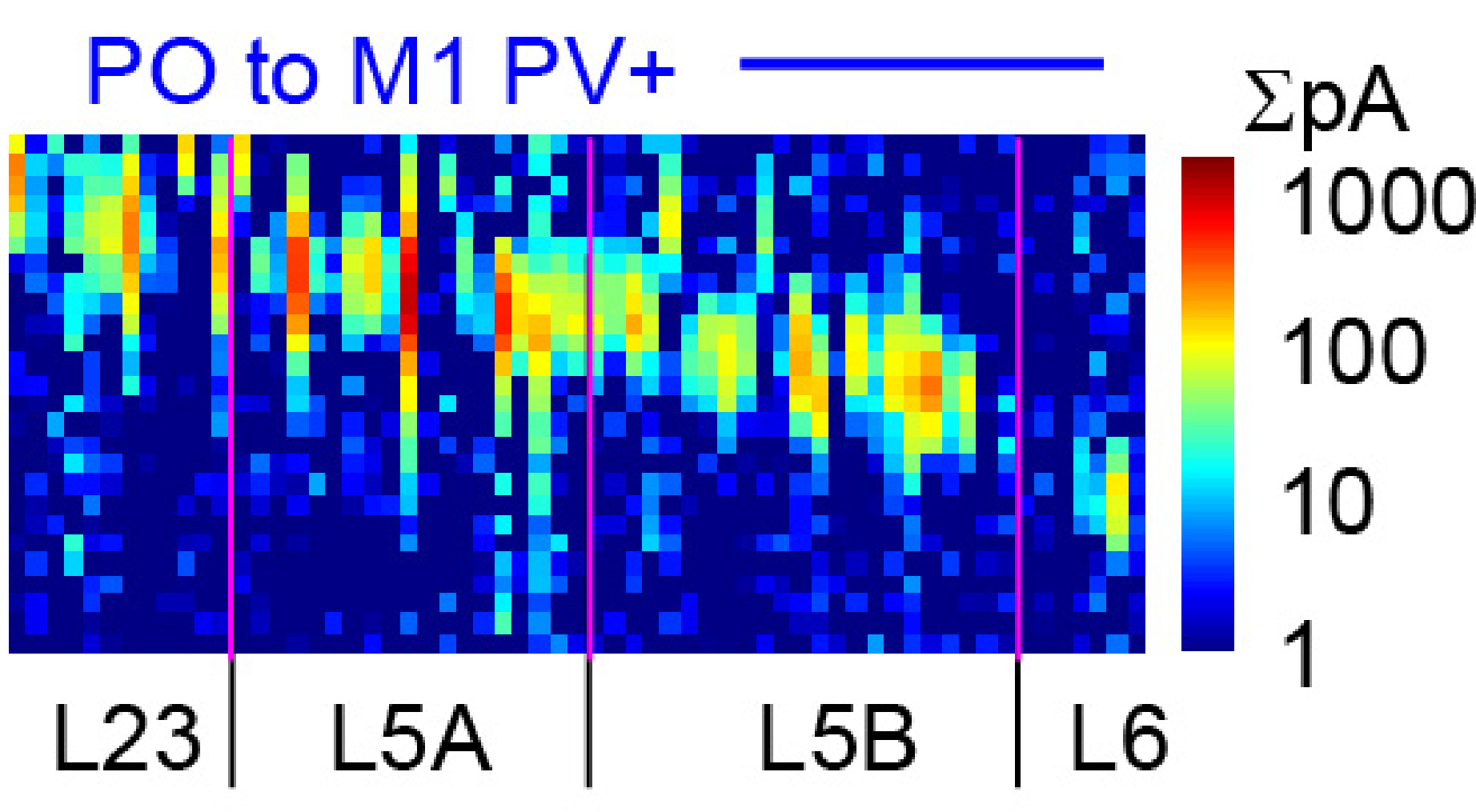

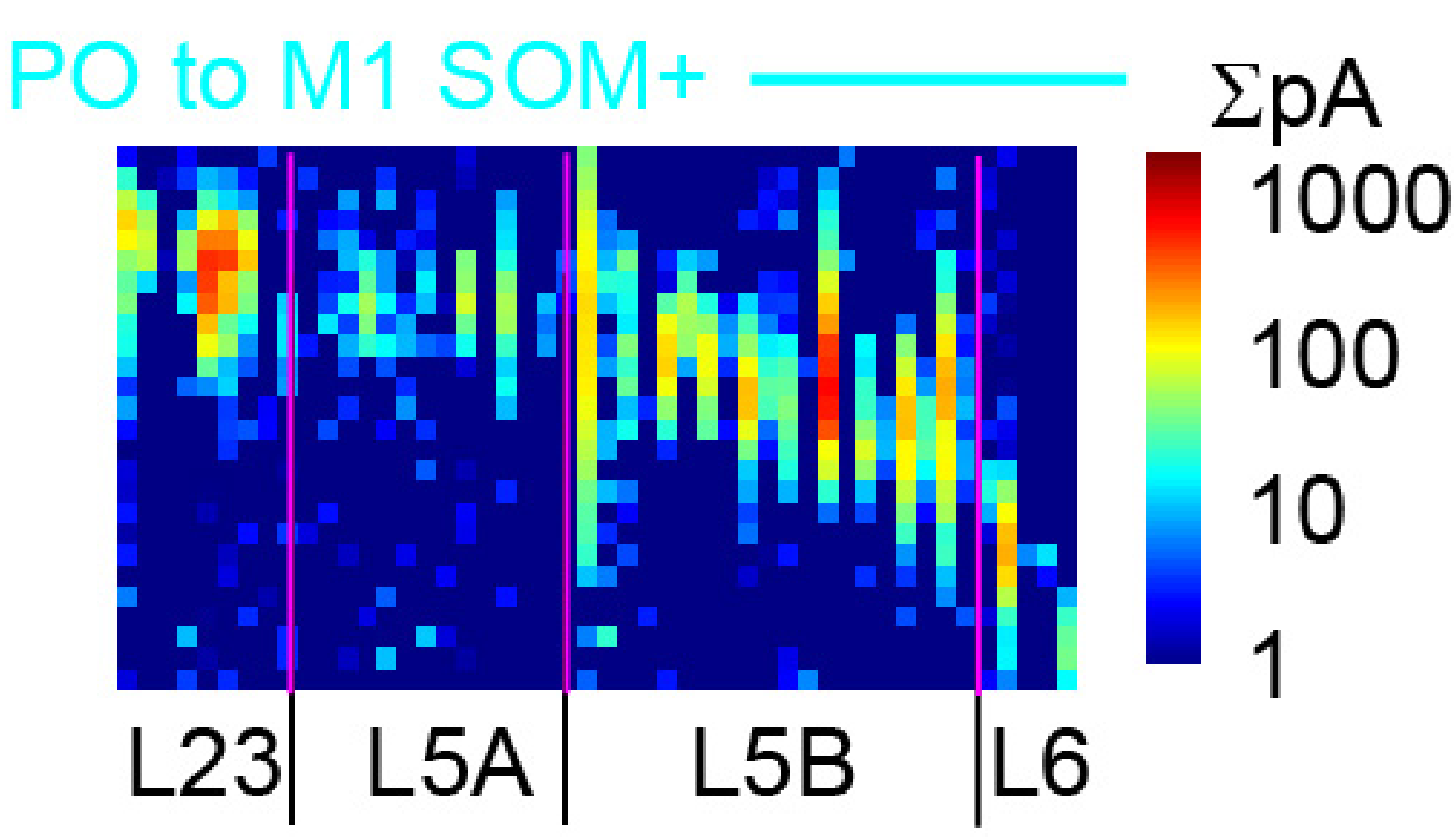

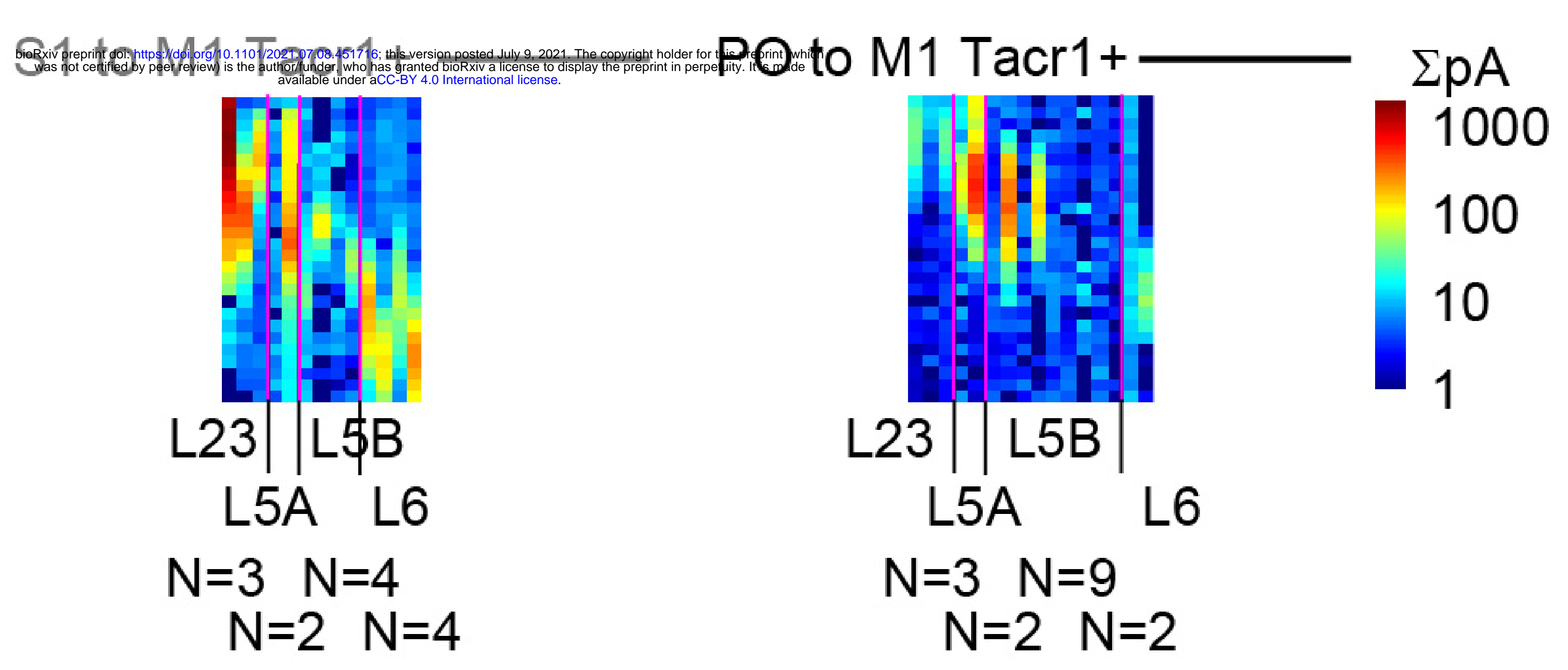

h Total Input to Interneurons

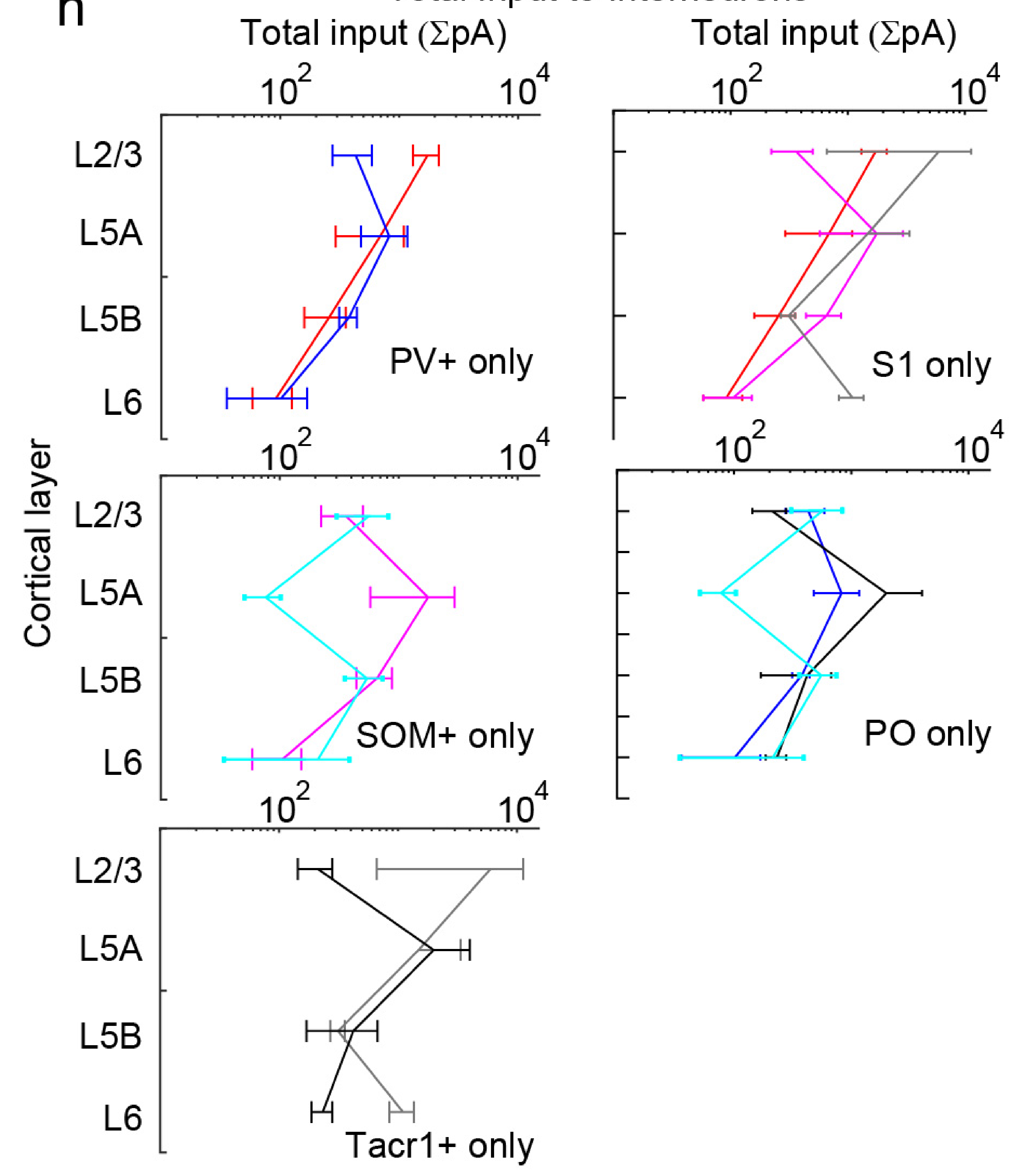

Figure 6 | Complementary patterns of input to specific interneuron types

(a-d) Summary graphs comparing the distribution of excitatory input to $P V+$ and $S O M+$ neurons. These plots compare normalized input from (a) $\mathrm{S} 1$ only or (b) PO only to PV+ and SOM+ neurons. (c-d) compare input to PV+ only (c) or SOM+ only interneurons. (e) The pattern of normalized input strength to pyramidal neurons (Pyr) from S1 and PO, after Mao et al (2011) and Hooks et al. (2013). (f) Cartoon summarizing input maps. (g)

Absolute input strength for $\mathrm{S} 1$ and $\mathrm{PO}$ inputs, plotted as input vectors on a log scale. Top row, PV+ neurons; middle row, SOM+ neurons, bottom row, Tacr1+ neurons (a subset of SOM+ neurons). Left column, S1 inputs. Right column, PO inputs. Data presented as in Figs 2-5. For Tacr1+ neurons, $\mathrm{N}=3 / 2 / 4 / 4$ for $\mathrm{S} 1$ input to $\mathrm{L} 2 / 3, \mathrm{~L} 5 \mathrm{~A}, \mathrm{~L} 5 \mathrm{~B}$, and $\mathrm{L6} ; \mathrm{N}=3 / 2 / 9 / 2$ for $\mathrm{PO}$ input. (h) Absolute input strength averaged by layer and plotted on a log scale for comparison between S1 and PO inputs to different interneuron types (left column) or input from S1 or PO (right column). 
a

S1 to M1 Pyr
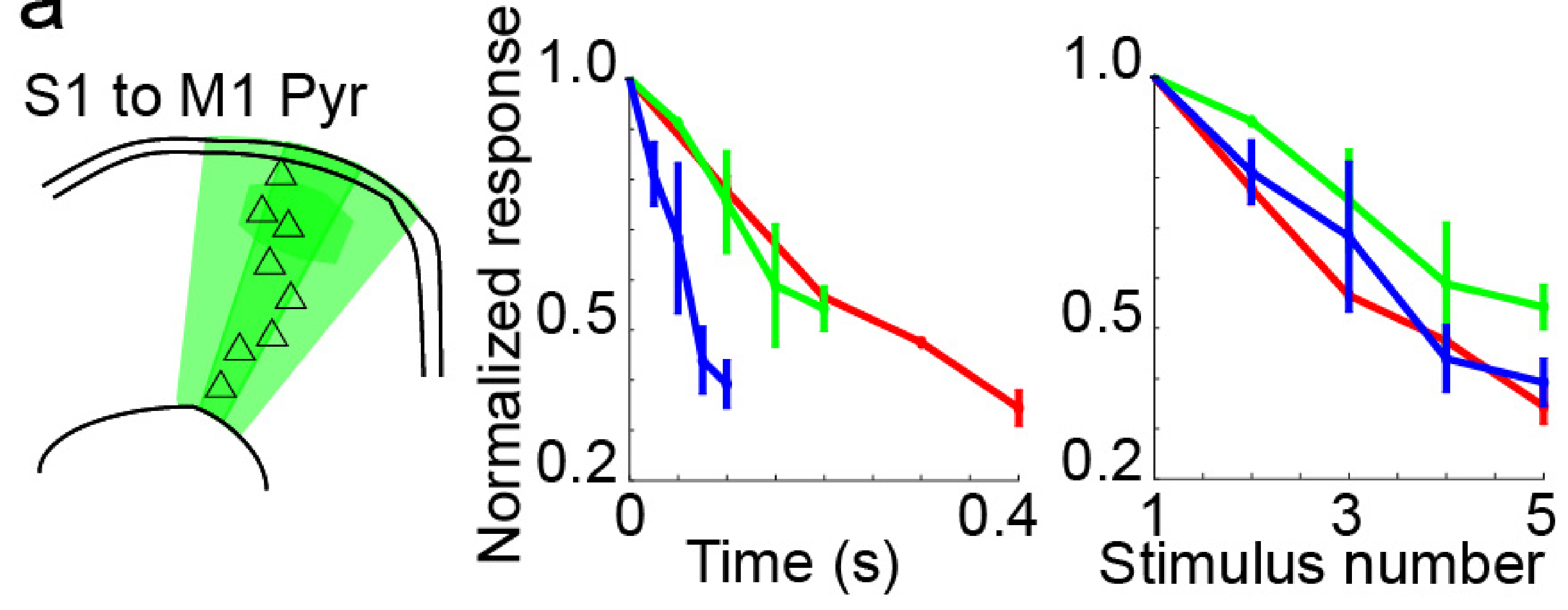

C

$\mathrm{S} 1$ to M1 PV+
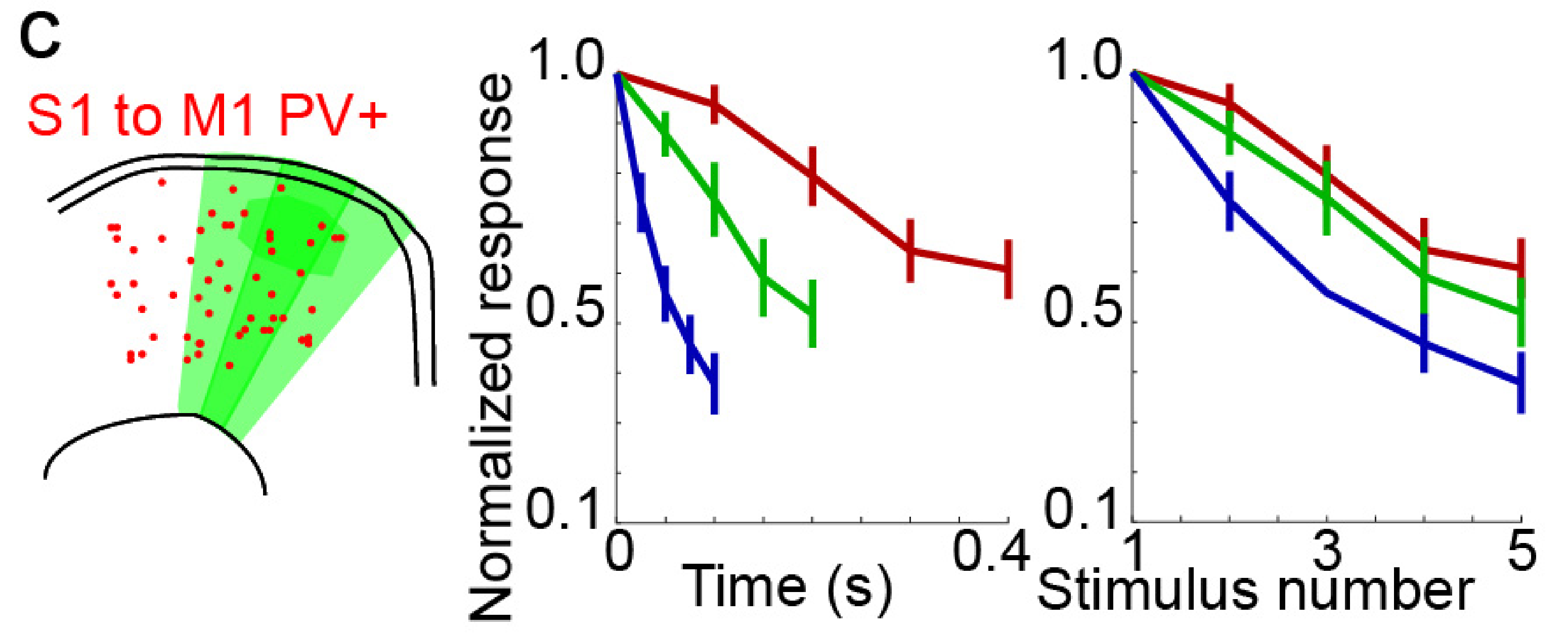

e

Comparison

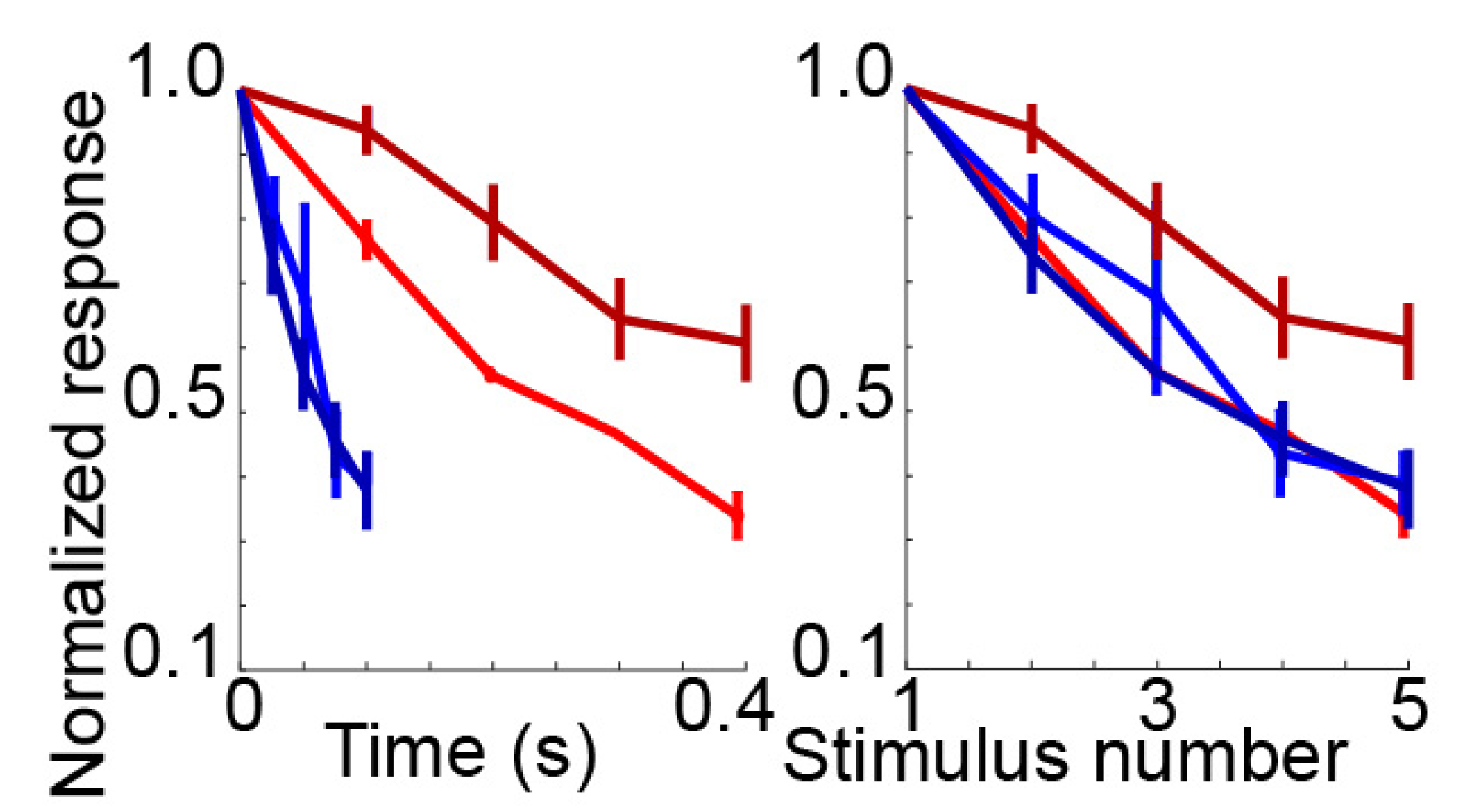

\section{PO to M1 Pyr}
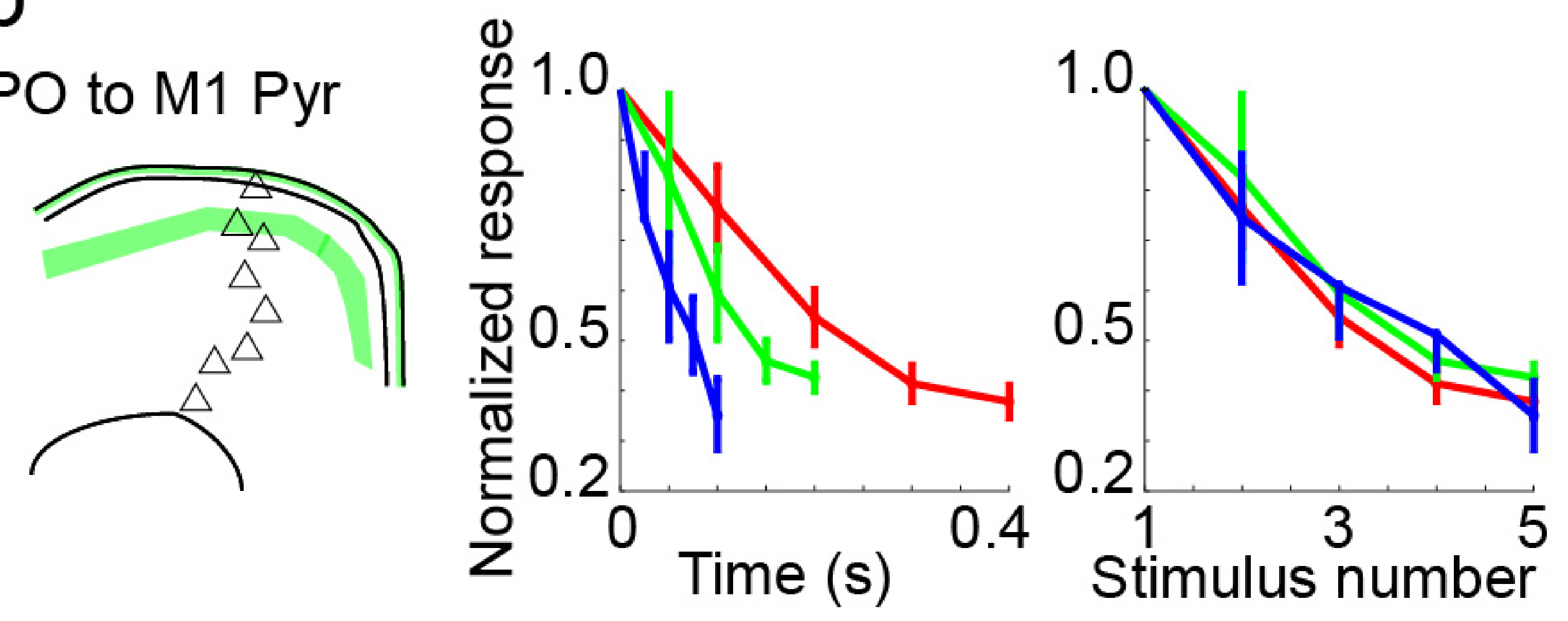

d

$\mathrm{PO}$ to $\mathrm{M} 1 \mathrm{PV}+$
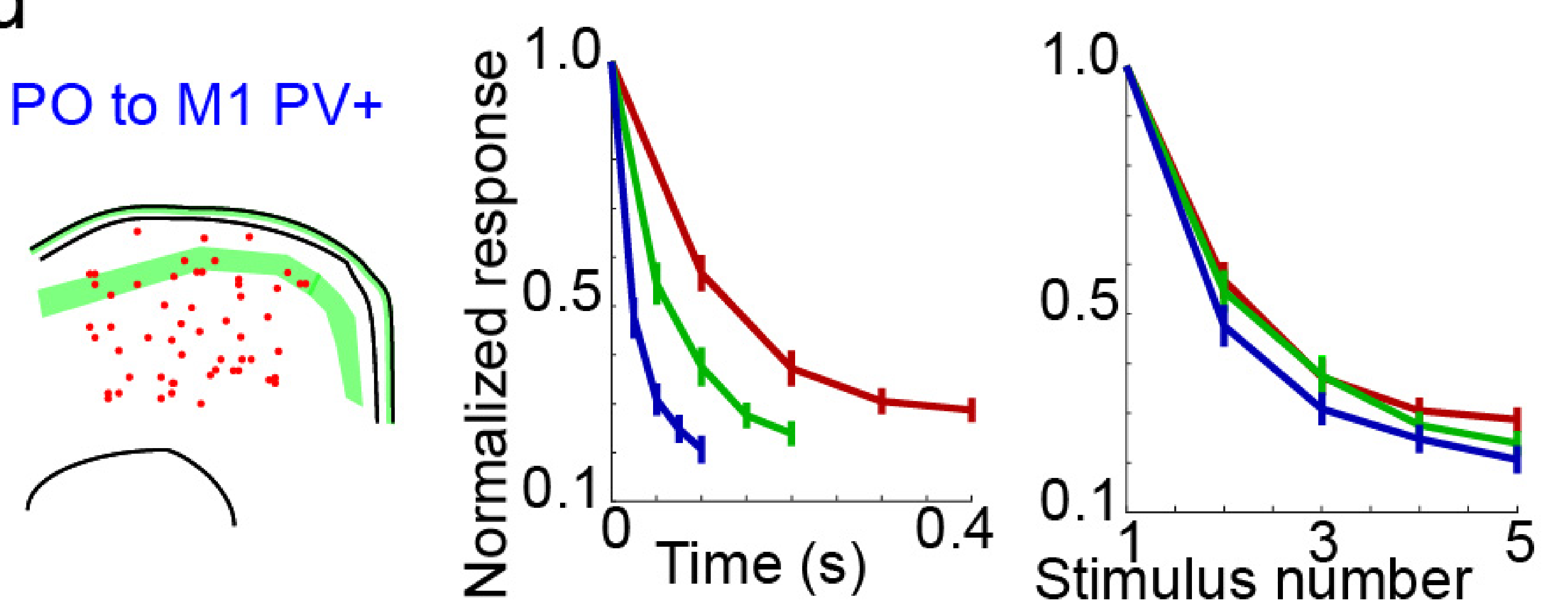

f

Comparison
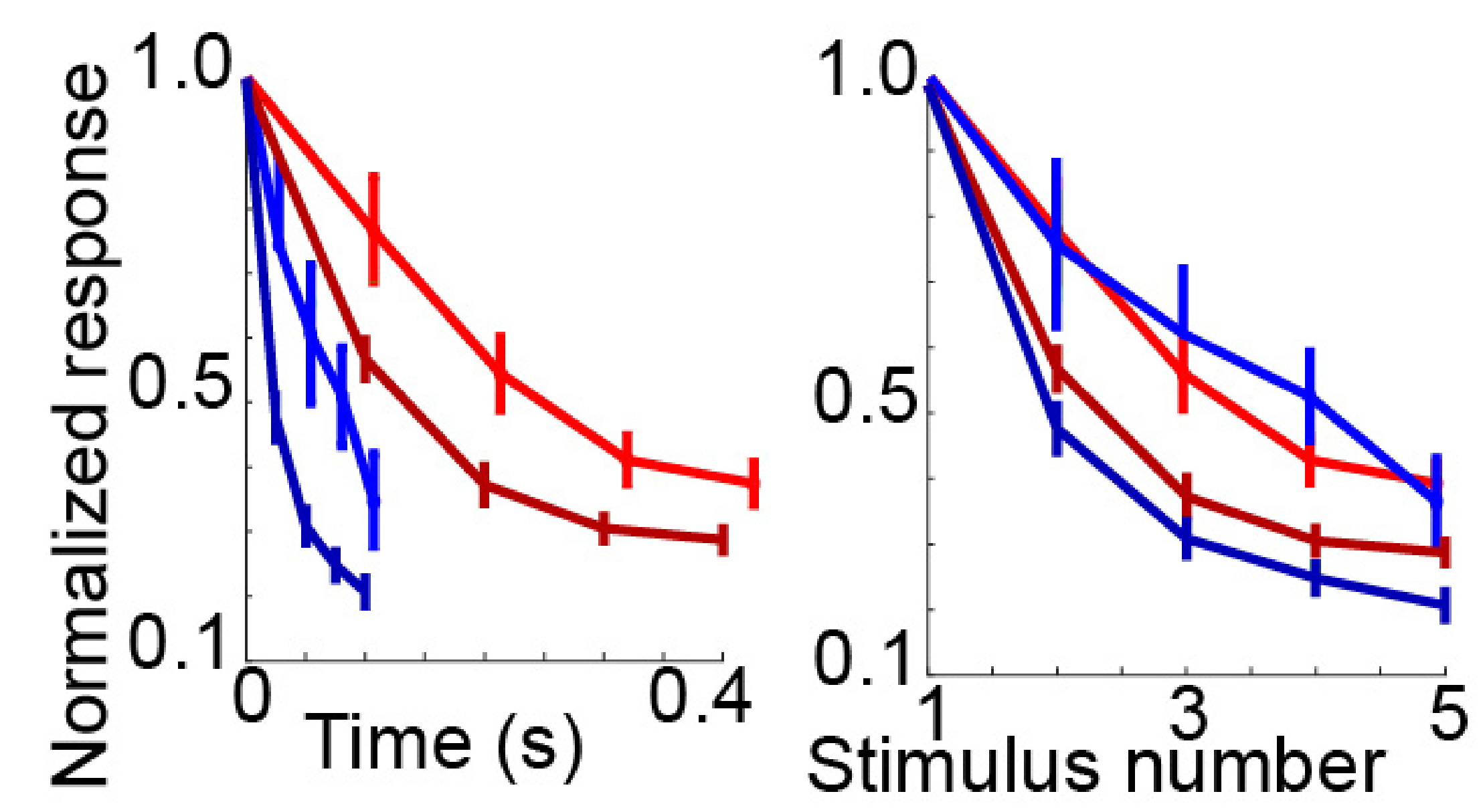

\section{Supplemental Figure 1 | Short-term plasticity comparisons of PV+ interneurons and pyramidal neurons}

(a) Short term plasticity of S1 input to pyramidal neurons. Left, normalized response to $\mathrm{S} 1$ stimulation at $10 \mathrm{~Hz}$ (red), $20 \mathrm{~Hz}$ (green), and $40 \mathrm{~Hz}$ (blue) stimulation of Chronos-GFP+. Summary graphs show responses plotted based on time of the pulses (left) or the stimulus number (right). (b) Short term plasticity of PO input to pyramidal neurons, plotted as in (a). (c,d) Short term plasticity of S1 input (c) and PO input (d) to PV+ interneurons plotted for comparison. (e,f) Comparison plot of the above data for $10 \mathrm{~Hz}$ and $40 \mathrm{~Hz}$ stimulation on the same axes. 
